\title{
4-Dimensional Black Holes from Kaluza-Klein Theories
}

\author{
Peter Breitenlohner ${ }^{1}$, Dieter Maison ${ }^{1}$, and Gary Gibbons ${ }^{2}$ \\ ${ }^{1}$ Max-Planck-Institut für Physik und Astrophysik, Werner-Heisenberg-Institut für Physik, P.O. \\ Box 401212, D-8000 München, Federal Republic of Germany \\ ${ }^{2}$ Department of Applied Mathematics and Theoretical Physics, Silver Street, Cambridge \\ CB3 9EW, England, and Laboratoire de Physique Théorique de l'Ecole Normale Supérieure*, \\ and Groupe d'Astrophysique Relativiste, Observatoire de Paris, Meudon, France
}

\begin{abstract}
In this paper we consider generalizations in 4 dimensions of the Einstein-Maxwell equations which typically arise from Kaluza-Klein theories. We specify conditions such that stationary solutions lead to non-linear $\sigma$-models for symmetric spaces. Using both this group theoretic structure and some properties of harmonic maps we are able to generalize many of the known existence and uniqueness theorems for black holes in Einstein-Maxwell theory to this more general setting.
\end{abstract}

\section{Introduction}

The advent of Einstein's non-linear theory of General Relativity seemed to open up the possibility of finding particle-like solutions of the classical equations of motion which are singularity free. However work by Serini [1], Einstein and Pauli [2], and Lichnerowicz [3] established that if a solution is asymptotically flat, topologically trivial and globally stationary (i.e. admits an everywhere time-like Killing vector field) then it must be flat space. In modern parlance one might say that Einstein's vacuum theory does not admit strictly stationary "soliton" solutions which are topologically trivial and without singularities.

In fact Lichernowicz's argument does extend to topologically non-trivial solutions of the form $\Sigma \times \mathbf{R}$, where $\Sigma$ is a complete asymptotically flat spacelike surface with perhaps more than one asymptotic region, but it was necessary for him to assume that the Killing vector is everywhere time-like. The possibility of more than one asymptotic region did not appear explicitly in his work, but in view of the "wormhole" structure of the constant time surfaces of the Schwarzschild solution discovered by Einstein and Rosen [4] it is evidently necessary to allow for this case. At the time Einstein and Rosen appeared to believe that they had obtained a singularity free solution, but as the global structure of the Schwarzschild solution became better understood it became clear that there was indeed a

\footnotetext{
* Laboratoire Propre du Centre Nationale de la Recherche Scientifique, associé à l'Ecole Normale Supérieure et à l'Université de Paris-Sud
} 
singularity, but not at the "throat" and not at the partial Cauchy surfaces but in their future and past. Furthermore the solution is not everywhere static.

During the late 50's work by the Princeton school [5] provided a better understanding of the possible topologically non-trivial initial data for Einstein's vacuum and Einstein-Maxwell theory, but the time evolution of such data remained obscure until the study of gravitational collapse in the 60's and 70's led to the realization that:

i) Space-time singularities seemed inevitably to arise during gravitational collapse but that there singularities appear to be hidden from infinity behind event horizons, inside what became known as "black holes".

ii) That the endpoint of the collapse is almost independent of the initial conditions - the only time independent final states being black hole solutions which are specified by just a few parameters - the mass and angular momentum in the vacuum case and in addition the electric and magnetic charges in the EinsteinMaxwell case.

By the time of the 1972 Les Houches Summer School [6] and certainly by the time of the Einstein centennial 1979 [7] there existed a chain of theorems and arguments which almost completely established that the most general asymptotically flat black hole solutions were either

i) the Kerr-Newman family [8] or

ii) the Papapetrou-Majumdar metrics [9].

Since 1979 there has been no fundamental change in this general picture but a number of new results have been obtained, the most important of which are

i) the proof of the "Positive Mass Theorem" [10];

ii) the completion by Bunting [11] and Mazur [12] of the programme initiated by Carter [13] of establishing the uniqueness of the Kerr-Newman solution amongst axisymmetric stationary solutions with a single horizon;

iii) the extension by Bunting and Masood-Ul-Alaam [14] of the original Israel theorem [15] to exclude multi-static vacuum black holes.

These papers are notable for the introduction of new techniques. Witten exploited concepts connected with supergravity, in particular the concept of a Killing spinor. Bunting and Mazur make use of the harmonic map and non-linear $\sigma$-model nature of the stationary field equations. The work of Bunting and Masood-Ul-Alaam makes essential use of the Positive Mass Theorem [10]. This theorem also allows a simple extension fo the Serini-Einstein-Pauli-Lichnerowicz theorem (see [16] and our Theorem 2.8).

The introduction of topological ideas into flat space field theory and in particular the notions of a "soliton" in the 70's prompted a reexamination of the "particle" concept in General Relativity and the extent to which black holes could be regarded as solitons [17]. Both classically and quantum mechanically a number of arguments were advanced suggesting that only the extreme ReissnerNordstrøm black holes (possibly magnetically rather than electrically charged) could be regarded as solitons. In particular in the absence of conserved vector charges there are no solitons. This view was strengthened and the links with other parts of soliton theory made closer with the construction of extended supergravity models. It was discovered that the charge in the Reissner-Nordstrøm solution is a "central charge" in the sense of supersymmetry and the inequality $|Q| \leqq \kappa M$ is a 
Bogomolny type inequality which is saturated only in the case that the solution (regarded as a solution of the supersymmetric extension of Einstein-Maxwell theory) is "supersymmetric", i.e. admits a Killing spinor $[18,19]$. This opens up the question of the soliton and black hole structure of supergravity theories. It soon becomes apparent that from the point of view of the purely bosonic fields the relevant field equations all have a very similar structure which is also shared by theories obtained by dimensional reduction from higher dimensional gravity theories with scalar and abelian vector fields. The scalar fields fall into a non-linear $\sigma$-model and have a rather simple coupling to the vector fields, the entire model exhibiting a large amount of on-shell and off-shell (duality type) symmetries. Further reduction to 3 dimensions increases the symmetry, since by dualization the vector fields may be replaced by scalar fields, and we obtain 3-dimensional gravity coupled to a large non-linear $\sigma$-model.

The symmetries may be used to generate whole families of new solutions from old ones and as such have been extensively studied [20-23]. It is the purpose of the present paper to combine the global approach based on uniqueness theorems with the solution generating technique based on symmetries of the local field equations to obtain a fairly complete picture of the black hole structure of the wide class of theories envisaged here.

A further link with soliton concepts was established with the discovery of the "complete integrability" of the 2-dimensional equations describing stationary, axially symmetric solutions of Einstein's vacuum respectively the EinsteinMaxwell theory $[24,25]$. Adaption of the "Inverse Scattering Method" and related techniques known from classical soliton equations like KdV or Sine-Gordon provided an effective new tool to produce hitherto unknown families of exact solutions with a 2-parameter abelian isometry group [26-28]. The extension of these techniques to the class of theories considered here will be reserved for a forthcoming publication [29].

The organization of the paper is as follows. In Sect. 2 we describe generalized Einstein-Maxwell systems in four space-time dimensions as they typically arise in Kaluza-Klein theories and discuss the concept of stationarity for its solutions. In Sect. 3 we show how the dimensional reduction to 3 dimensions - relevant for stationary configurations - leads to non-linear $\sigma$-models for symmetric spaces $G / H$ and discuss some group theoretical points connected with their particular structure. Furthermore we construct the 2-dimensional theories describing stationary, axially symmetric solutions.

Section 4 is devoted to stationary, spherically symmetric solutions given in terms of geodesics of $G / H$. In particular the conditions for these solutions to represent black holes are analyzed. Section 5 contains the boundary conditions for general black holes, whereas in Sects. 6 and 7 we generalize known existence and uniqueness theorems (No Hair theorems etc.) for static respectively rotating black holes.

In Sect. 8 we try to give a picture of time-independent solutions describing several black holes in equilibrium. In view of the fact that very little is still known about these very interesting configurations our discussion remains necessarily somewhat sketchy. 
Finally in Sect. 9 we list some open problems accumulated in the course of our attempt to present a comprehensive picture of black holes from Kaluza-Klein theories.

Appendix A contains fundamentals and notation about non-compact Riemannian and pseudo-Riemannian symmetric spaces. In Appendix B we give a complete list of non-linear $\sigma$-models arising from the theories discussed in Sects. 2 and 3 and some examples for the static truncation of such $\sigma$-models.

\section{Description of the 4-Dimensional Theory}

Inspired by the experience from Kaluza-Klein respectively extended supergravity theories we shall study field theories (in $3+1$ space-time dimensions) with massless scalar and vector fields coupled to gravity where the scalars form a $\bar{G} / \bar{H}$ non-linear $\sigma$-model and the field strengths of the vectors transform with some representation of the global (constant parameter) symmetry group $\bar{G}$.

In particular we are interested in stationary solutions, i.e. solutions allowing for a time-like Killing vector field. For those we obtain, after suitable duality transformations, a dimensionally reduced theory with scalars coupled to gravity in 3 space dimensions. Sometimes (e.g. stationary, axisymmetric solutions) it is also convenient to perform the reduction to 3 dimensions for a space-like Killing vector field leading to a theory in $2+1$ space-time dimensions. In the stationary case the scalar fields of this dimensionally reduced theory are the original scalar fields from the $\bar{G} / \bar{H} \sigma$-model, two scalar fields from gravity (the norm $\Delta$ of the Killing vector playing the role of a gravitational potential and the twist or NUT potential $\tilde{\psi}$ ) and two scalars (electric and magnetic potential) from each vector field. It is well known that the two scalars from gravity form an $S L(2) / S O(2) \sigma$-model [22]; we are, however, interested in those cases where the resulting 3 dimensional theory has a much larger symmetry such that all scalars form one $G / H \sigma$-model.

The coset space $\bar{G} / \bar{H}$ should be a non-compact Riemannian symmetric space [30], i.e. $\bar{G}$ is a non-compact Lie group and $\bar{H}$ its maximal compact subgroup. (The trivial case that $\bar{G}=\bar{H}$ is compact leads to a trivial $\sigma$-model with no scalars, but nevertheless the action of the group $\bar{G}$ on the vectors is of interest.) Furthermore the coupling to the vector fields should be such that the resulting energy density $T_{00}$ is positive definite. A space-like Killing vector field will then lead to a $G / H^{\prime}$ $\sigma$-model in $2+1$ dimensions with $G / H^{\prime}$ again a non-compact Riemannian symmetric space. A time-like Killing vector field yields a $G / H \sigma$-model in 3 dimensions with the same group $G$, but $H$ is a non-compact version of $H^{\prime}$ (which was the maximal compact subgroup of $G$ ) such that $G / H$ is a symmetric space with indefinite metric where the signature of those scalars which arose from vector fields has been changed.

This situation is illustrated with a few examples in Table 1 (some of them are the bosonic sector of extended supergravity theories).

\subsection{Non-Linear $\sigma$-Models Coupled to Vector Fields and Gravity}

Consider a Lagrangian field theory over a space-time manifold $\Sigma$ with coordinates $x^{\alpha}$ and metric $g_{\alpha \beta}(x)$ and fields with values in a target space $\bar{\Phi}$ with coordinates $\bar{\phi}^{i}$ 
Table 1. Some examples for non-linear $\sigma$-models in three dimensions

\begin{tabular}{|c|c|c|c|}
\hline$G / H^{\prime}$ & $G / H$ & $\bar{G} / \bar{H}$ & $3+1$ dimensional theory \\
\hline$S L(n+2)$ & $S L(n+2)$ & \multirow{2}{*}{$G L(n) / S O(n)$} & $n+4$ dimensional \\
\hline$S O(n+2)$ & $S O(n, 2)$ & & $\begin{array}{l}\text { Einstein gravity with } \\
n \text { Killing vectors }\end{array}$ \\
\hline$S U(2,1)$ & $S U(2,1)$ & \multirow{2}{*}{$U(1) / U(1)$} & \multirow{2}{*}{$\begin{array}{l}\text { Einstein-Maxwell } \\
\qquad(N=2 \text { supergravity) }\end{array}$} \\
\hline$\overline{S(U(2) \times U(1))}$ & $\overline{S(U(1,1) \times U(1))}$ & & \\
\hline $\operatorname{SO}(8,2)$ & $S O(8,2)$ & $S O(6) \times S O(2,1)$ & \multirow{2}{*}{$N=4$ supergravity } \\
\hline$\overline{S O(8) \times S O(2)}$ & $\overline{S O(6,2) \times S O(2)}$ & $S O(6) \times S O(2)$ & \\
\hline$S O(8,8)$ & $S O(8,8)$ & $S O(6,6) \times S O(2,1)$ & \multirow{2}{*}{$\begin{array}{r}N=4 \text { supergravity } \\
\quad+\text { supersym. Maxwell } \\
\text { (10 dim. supergravity) }\end{array}$} \\
\hline$\overline{S O}(8) \times S O(8)$ & $\overline{S O(6,2) \times S O(2,6)}$ & $\overline{S O(6) \times S O(6) \times S O(2)}$ & \\
\hline$E_{8(+8)} / S O(16)$ & $E_{8(+8)} / S O^{*}(16)$ & $E_{7(+7)} / S U(8)$ & $\begin{array}{l}N=8 \text { supergravity } \\
\quad(11 \text { dim. supergravity) }\end{array}$ \\
\hline
\end{tabular}

and metric $\bar{\gamma}_{i j}$. The action for such a non-linear $\sigma$-model coupled to gravity

$$
S_{\bar{\Phi}}=\int_{\Sigma} \sqrt{g} d x\left(-\frac{1}{2} R(x)+\frac{1}{2} g^{\alpha \beta}(x) \partial_{\alpha} \bar{\phi}^{i}(x) \partial_{\beta} \bar{\phi}^{j}(x) \bar{\gamma}_{i j}(\bar{\phi}(x))\right)
$$

leads to the field equations

$$
\begin{gathered}
R_{\alpha \beta}-\partial_{\alpha} \bar{\phi}^{i} \partial_{\beta} \bar{\phi}^{j} \bar{\gamma}_{i j}(\bar{\phi})=0, \\
D^{\alpha} \partial_{\alpha} \bar{\phi}^{i}(x)=0 .
\end{gathered}
$$

The solutions of Eq. (2.3) are harmonic maps from $\Sigma$ (with metric $g$ ) to $\bar{\Phi}$ (with metric $\bar{\gamma}$ ). We are interested in particular in the case where $\bar{\Phi}$ is a non-compact Riemannian symmetric space $\bar{G} / \bar{H}[30,31]$. Using the notation of Appendix A, which contains a description of non-linear $\sigma$-models based on symmetric spaces, we can rewrite Eqs. (2.1-3) in the form [compare Eqs. (A6, 7, 11, 13)]

$$
\begin{gathered}
S_{\bar{G} / \bar{H}}=\int_{\Sigma} \sqrt{g} d x\left(-\frac{1}{2} R+\frac{1}{2} g^{\alpha \beta}\left\langle\bar{J}_{\alpha}, \bar{J}_{\beta}\right\rangle\right), \\
R_{\alpha \beta}-\left\langle\bar{J}_{\alpha}, \bar{J}_{\beta}\right\rangle=0 \\
D^{\alpha} \bar{J}_{\alpha}=0
\end{gathered}
$$

where $\bar{J}=\frac{1}{2} \bar{M}^{-1} \partial \bar{M}$.

We want to add vector fields to the action (2.1) in such a way that the resulting field equations are $\bar{G}$-invariant if the (abelian) field strengths together with their duals are transformed with a suitable representation $\bar{\varrho}$ of $\bar{G}$. The situation is somewhat complicated by the fact that the action of $\bar{G}$ may involve duality transformations (e.g. $U(1)$ for Einstein-Maxwell) which transform solutions of the field equations into solutions but which are not symmetries of the action ("onshell" symmetries). 
Let there be a $k$-dimensional column $B_{\alpha}=\left(B_{\alpha}^{\mathrm{I}}\right)(I=1, \ldots, k)$ of real vector fields with field strengths $G_{\alpha \beta}=\partial_{\alpha} B_{\beta}-\partial_{\beta} B_{\alpha}$ and their duals ${ }^{*} G^{\alpha \beta}=\frac{1}{2 \sqrt{g}} \varepsilon^{\alpha \beta \gamma \delta} G_{\gamma \delta}$ $\left({ }^{* *} G=-G\right)$ satisfying Bianchi identities $\nabla_{\alpha}^{*} G^{\alpha \beta}=0$. The most general gauge invariant action quadratic in the field strengths is

$$
S_{V}=\int_{\Sigma} \sqrt{g} d x\left(-\frac{1}{4} G_{\alpha \beta}^{T}\left(\mu(\bar{\phi}) G^{\alpha \beta}-v(\bar{\phi})^{*} G^{\alpha \beta}\right)\right),
$$

where $\mu=\left(\mu_{I J}\right)$ and $v=\left(v_{I J}\right)$ are real symmetric matrices depending on the fields $\bar{\phi}^{i}$ parametrizing the coset space $\bar{G} / \bar{H}$. This action yields field equations

$$
\nabla_{\alpha}\left(\mu G^{\alpha \beta}-v^{*} G^{\alpha \beta}\right)=0,
$$

which can be interpreted [32] as Bianchi identities $\nabla_{\alpha}^{*} H^{\alpha \beta}=0$ for field strengths $H_{\alpha \beta}=\partial_{\alpha} C_{\beta}-\partial_{\beta} C_{\alpha}$ derived from dual potentials $C_{\alpha}=\left(C_{\alpha I}\right)$. We now have $2 k$ vector fields $A_{\alpha}=\left(\begin{array}{l}B_{\alpha} \\ C_{\alpha}\end{array}\right)$ and field strengths $F_{\alpha \beta}=\partial_{\alpha} A_{\beta}-\partial_{\beta} A_{\alpha}$ satisfying the linear relation

with

$$
F_{\alpha \beta}=\bar{Y} \hat{\bar{M}}^{*} F_{\alpha \beta}
$$

$$
\bar{Y}=\left(\begin{array}{cc}
0 & \eta^{T} \\
-\eta & 0
\end{array}\right), \quad \hat{\bar{M}}=\left(\begin{array}{cc}
\mu+v \mu^{-1} v & v \mu^{-1} \eta^{-1} \\
\left(\eta^{T}\right)^{-1} \mu^{-1} v & \left(\eta^{T}\right)^{-1} \mu^{-1} \eta^{-1}
\end{array}\right),
$$

where $\eta$ is some real $k \times k$ matrix and the symmetric matrix $\hat{M}$ satisfies $\bar{Y} \hat{M} \bar{Y}$ $=-\hat{M}^{-1}$. The matrix $\eta$ is, of course, quite unnecessary at this point, but will become useful if we want the group elements $P(x) \in \bar{G}$ parameterizing the coset space $\bar{G} / \bar{H}$ (compare Appendix A) to be represented by triangular matrices. The equations

$$
\nabla_{\alpha}^{*} F^{\alpha \beta}=-\nabla_{\alpha}\left(\bar{Y} \hat{M} F^{\alpha \beta}\right)=0
$$

can be interpreted as field equations and Bianchi identities for either $G_{\alpha \beta}$ or $H_{\alpha \beta}$. These equations are $\bar{G}$-invariant under the transformation

$$
\bar{G} \ni \bar{g}: F_{\alpha \beta} \rightarrow \bar{\varrho}(\bar{g}) F_{\alpha \beta},
$$

provided the real representation $\bar{\varrho}$ satisfies $\varrho\left(\bar{g}^{-1}\right)^{T}=\bar{Y}^{-1} \bar{\varrho}(\bar{g}) \bar{Y}$ and we can construct a matrix $\hat{\bar{M}}(\bar{\phi})$ of the form (2.7) depending on the scalars $\bar{\phi}^{i}$ in such a way that

$$
\bar{G} \ni \bar{g}: \hat{\bar{M}}(\bar{\phi}) \rightarrow \bar{\varrho}\left(\bar{g}^{-1}\right)^{T} \hat{\bar{M}}(\bar{\phi}) \varrho\left(\bar{g}^{-1}\right) .
$$

The contribution from $S_{V}$ to the stress tensor

$$
T_{\alpha \beta}^{(V)}=-G_{\alpha \gamma}^{T} \mu G_{\beta}{ }^{\gamma}+\frac{1}{4} g_{\alpha \beta} G_{\gamma \delta}^{T} \mu G^{\nu \delta}=-\frac{1}{2} F_{\alpha \gamma}^{T} \hat{\bar{M}} F_{\beta}{ }^{\gamma}=-\frac{1}{2} F_{\alpha \gamma}^{T} \bar{Y}^{*} F_{\beta}{ }^{\gamma},
$$

as well as the contribution to the scalar field equations

$$
\frac{\delta S_{V}}{\delta \bar{\phi}^{i}}=-\frac{1}{4} \sqrt{g} G_{\alpha \beta}^{T}\left(\frac{\delta \mu}{\delta \bar{\phi}^{i}} G^{\alpha \beta}-\frac{\delta v}{\delta \bar{\phi}^{i}} * G^{\alpha \beta}\right)=-\frac{1}{8} \sqrt{g} F_{\alpha \beta}^{T} \frac{\delta \hat{\bar{M}}}{\delta \bar{\phi}^{i}} F^{\alpha \beta}
$$

will then be explicitly $\bar{G}$-invariant. Since $T_{00}^{(V)}$ must be positive for a physically meaningful theory, the matrix $\hat{M}$ (and hence $\mu$ ) must be positive definite. 
In order to construct such an $\hat{\bar{M}}$ consider a (not necessarily irreducible) $2 k$ dimensional real matrix representation $\bar{\varrho}$ of $\bar{G}$

$$
\bar{\varrho}: \bar{G} \ni \bar{g} \rightarrow \hat{\bar{g}} \equiv \varrho(\bar{g})
$$

such that $\left(\hat{\bar{g}}^{-1}\right)^{T}=\bar{Y}^{-1} \hat{\bar{g}} \bar{Y}$ and

$$
\widehat{\bar{\tau}(\bar{g})}=\bar{X}^{-1}\left(\hat{\bar{g}}^{-1}\right)^{T} \bar{X}=\bar{X}^{-1} \bar{Y}^{-1} \hat{\bar{g}} \bar{Y} \bar{X}
$$

with some positive definite real symmetric matrix $\bar{X}$ satisfying $\bar{Y} \bar{X} \bar{Y}=-\bar{X}^{-1}$. Given $\bar{P} \in \bar{G}$ and $\bar{\varrho}(\bar{P})=\hat{\hat{P}}$, we find $\bar{\varrho}(\bar{M})=\bar{X}^{-1} \hat{\bar{M}}$, where $\hat{\bar{M}}$ is the real symmetric matrix $\hat{P}^{T} \bar{X} \hat{P}$ transforming as required by Eq. (2.10). Furthermore $\bar{Y} \hat{\bar{M}} \bar{Y}=-\hat{\bar{M}}^{-1}$, and therefore $\hat{\bar{M}}$ has exactly the structure (2.7) with real symmetric $k \times k$ matrices $\mu$ and $\nu$.

Collecting all the terms in the Lagrangian and the field equations describing the coupling of the gravitational field $g_{\alpha \beta}$, the vector field strengths and their duals comprised in $F_{\alpha \beta}$ and the scalars $\bar{\phi}^{i}$ parametrizing $\bar{M}$, we obtain [using Eq. (A.11)]

$$
\begin{gathered}
\mathscr{L}^{(4)}=\sqrt{g}\left(-\frac{1}{2} R^{(4)}+\frac{1}{8} g^{\alpha \beta}\left\langle\bar{M}^{-1} \partial_{\alpha} \bar{M}, \bar{M}^{-1} \partial_{\beta} \bar{M}\right\rangle-\frac{1}{4} G_{\alpha \beta}^{T}\left(\mu(\bar{\phi}) G^{\alpha \beta}-v(\bar{\phi})^{*} G^{\alpha \beta}\right)\right) \\
R_{\alpha \beta}=-\frac{1}{2} F_{\alpha \gamma}^{T} \hat{\bar{M}} F_{\beta}^{\gamma}+\frac{1}{4}\left\langle\bar{M}^{-1} \partial_{\alpha} \bar{M}, \bar{M}-1 \partial_{\beta} \bar{M}\right\rangle \\
\nabla_{\alpha}\left(\hat{\bar{M}} F^{\alpha \beta}\right)=0 \\
\nabla^{\alpha}\left(\bar{M}^{-1} \partial_{\alpha} \bar{M}\right)=-\frac{1}{2 \hat{\bar{c}}}\left(F^{\alpha \beta} F_{\alpha \beta}^{T} \hat{\bar{M}}\right)_{\mathrm{pr}}
\end{gathered}
$$

[see Eq. (A.8) for the definition of $(\ldots)_{\mathrm{pr}}$ ]. Let us finally remark that these field equations are scale invariant if we assign scaling dimension one to $g_{\alpha \beta}$ and zero to $A_{\alpha}$ and $\bar{\phi}^{i}$.

\subsection{Stationarity and Staticity}

In this article we shall consider only stationary space-times which are asymptotically flat (or possibly asymptotically Taub-NUT). In addition we require that the matter fields tend asymptotically to the "classical vacuum" configuration, i.e. the scalar fields tend to constants and the vector field strengths to zero. A detailed discussion of the asymptotic conditions for the Einstein-Maxwell theory which can be readily generalized to our case can be found in [33]. Furthermore we shall assume that the space-times considered fulfill the standard causality requirements (compare [34] for a precise formulation).

There are two different definitions of metric stationarity, a weaker and a stronger notion, which are occasionally confused. Moreover, since in addition to the metric we are concerned with scalar fields and vector field strengths, there are many different possible notions of stationarity and staticity. We shall now define the notions of stationarity and staticity used in this paper. Let us begin with (strict) metric stationarity.

Definition 2.1 (Metric Stationarity). A space-time manifold is said to be stationary if it admits a Killing vector field $K$ which is time-like near infinity.

Definition 2.2 (Strict Metric Stationarity). A stationary space-time is said to be strictly stationary if $K$ is everywhere time-like. 
Definition 2.3 (Stationary Configurations). A field configuration is called (strictly) stationary if the metric is (strictly) stationary and the Lie derivative with respect to $K$ of the scalar fields and the vector field strengths vanishes.

Even if the Einstein equations hold, it is not necessarily the case that stationarity of the metric implies stationarity of the configuration. Counterexamples for scalar fields are given in [35] and for vector fields in [36].

Definition 2.4 (Metric Staticity). A (strictly) stationary space-time is said to be (strictly) static if the Killing vector field $K$ is hypersurface orthogonal, i.e. if $K_{[\alpha ; \beta} K_{\gamma]}=0$.

Definition 2.5 (Ricci Tensor Staticity). A stationary space-time is said to have a static Ricci tensor if $K^{\gamma} R_{\gamma[\alpha} K_{\beta]}=0$.

Remark. It can be shown by purely local arguments that a static metric has a static Ricci tensor [13]. The converse would require a global "Lichnerowicz type" argument.

In order to define staticity for the scalar and vector fields it is necessary to assign each field a time reflection parity such that the action (2.15) is time reflection invariant. This assignment is in general not unique. Consider, e.g., the EinsteinMaxwell system: the electromagnetic field $F_{\alpha \beta}$ is usually considered as temporal vector field strength $F^{(+)}$, i.e. the magnetic field $B_{\alpha}={ }^{*} F_{\alpha \beta} K^{\beta}$ vanishes for a static solution; due to the duality invariance of the field equations $F_{\alpha \beta}$ could as well be considered as temporal pseudo vector field strength $F^{(-)}$with the consequence that a solution would be called static if the electric field $E_{\alpha}=F_{\alpha \beta} K^{\beta}$ vanishes.

Definition 2.6 (Strong Staticity). A field configuration consisting of a metric, temporal scalar fields $\phi^{(+)}$, temporal pseudo scalars $\phi^{(-)}$, temporal vector field strengths $F^{(+)}$and temporal pseudo vector field strengths $F^{(-)}$is said to be strongly static if the metric is static and

1. $\phi^{(-)}=0$,

2. $F_{[\alpha \beta}^{(+)} K_{\gamma]}=0$ and

3. ${ }^{*} F_{[\alpha \beta}^{(-)} K_{\gamma]}=0$.

Remark. Given a strongly static field configuration we can obtain by the action $(2.9,10)$ of the group $\bar{G}$ new configurations with a static metric which will in general not be strongly static.

Definition 2.7 (Static Subgroup of $\bar{G}$ ). The subgroup $\bar{G}_{\mathrm{st}} \subset \bar{G}$ preserving conditions 1,2 and 3 above will be called the static subgroup of $\bar{G}$.

Just as in Maxwell theory stationarity allows the introduction of scalar potentials, so for a stationary configuration of the type of theory considered we can introduce time-independent electric and magnetic scalar potentials $A$,

$$
F_{\alpha \beta} K^{\beta}=\partial_{\alpha} A .
$$

Note that $A$ contains electric and magnetic potentials since $F_{\alpha \beta}$ comprises both $G_{\alpha \beta}$ and ${ }^{*} G_{\alpha \beta}$ [compare Eq. (2.6)]. In a gauge in which the Lie derivative of the vector potentials $A_{\alpha}$ vanishes the scalar potentials $A$ are the time-components $K^{\alpha} A_{\alpha}$ of the vector potentials. 
We are now in a position to prove a generalization of the result of Serini, Einstein, Pauli and Lichnerowicz ruling out non-trivial stationary asymptotically flat and singularity free configurations.

The mass of any stationary asymptotically flat configuration is given by the Komar integral

$$
m=\frac{1}{8 \pi} \oint_{\infty} K^{\alpha ; \beta} d \Sigma_{\alpha \beta},
$$

where the integral is taken over any space-like 2 -sphere at infinity. Similarly the electric and magnetic charges may be expressed as

$$
Q_{A}=\frac{1}{8 \pi} \oint_{\infty} \hat{\bar{M}} F^{\alpha \beta} d \Sigma_{\alpha \beta} .
$$

Stationarity and use of the field equations allows one to derive the identity

$$
K_{; \beta}^{\alpha ; \beta}=-R^{\alpha \beta} K_{\beta}=-\frac{1}{2}\left(A^{T} \hat{\bar{M}} F^{\alpha \beta}\right)_{; \beta} .
$$

Theorem 2.8 (Generalized Serini-Einstein-Pauli-Lichnerowicz Theorem). A stationary solution of Eqs. (2.16-18) with an asymptotically flat globally hyperbolic space-time $\Sigma$, whose Cauchy surfaces are complete with a single asymptotic region and for which

(a) $\Sigma$ is Ricci-flat or

(b) the Cauchy surfaces are simply connected is empty Minkowski space.

Proof. To begin with we integrate Maxwell's Eq. (2.8) over a Cauchy surface and use Stokes' theorem to deduce that the charges $Q_{A}$ vanish. If the Cauchy surfaces and thus $\Sigma$ are simply connected the scalar potentials $A$ are globally defined. We can integrate the identity (2.22) over a Cauchy surface and use Stokes' theorem again to obtain the mass $m$ of the space-time. In case (a) the mass vanishes immediately. In case (b) we get a formula in terms of the charges $Q_{A}$ and the asymptotic values of the potentials

$$
m=-\frac{1}{2} A_{0}^{T} Q_{A} .
$$

Thus in both cases the mass vanishes. Since the stress tensor satisfies the dominant energy condition we may use the "Positive Mass Theorem" [10] to deduce that space-time is flat, the scalar fields are constant and the vector field strengths vanish.

Remark. Even in case (a) our theorem is stronger than the original result of Lichnerowicz [3] who assumed strict stationarity. This is possible because we used a 4-dimensional formulation and the "Positive Mass Theorem". The result rules out the existence of ergoregions without black holes or singularities.

Remark. The assumption of simple connectedness in case (b) is in fact quite reasonable since Gannon [37] has shown that a null-geodesically complete asymptotically flat space-time cannot have non-simply connected global Cauchy surfaces. 


\section{Dimensional Reduction}

In the following we shall consider only stationary respectively stationary, axisymmetric field configurations. For those it is possible to reformulate the 4-dimensional field theory described in Sect. 2 in terms of fields defined on the 3respectively 2-dimensional space of orbits $\Sigma_{3}$ respectively $\Sigma_{2}$ of the corresponding isometry group acting on $\Sigma$. This procedure is quite analogous to the "dimensional reduction" technique for Kaluza-Klein theories and will be described in the two subsequent sections.

\subsection{Dimensional Reduction from 4 to 3 Dimensions}

In this section we shall demonstrate how field configurations allowing one Killing vector field give rise to a "dimensionally reduced" 3-dimensional field theory. Since this reduction can be performed irrespective of the signature of the Killing vector field it can be applied to time-translations as well as axial rotations, but for reasons of simplicity we shall use the notations for time-translations. In case we want to explicitly refer to axial rotations we shall indicate this with a prime on the corresponding field.

For a field configuration allowing a Killing vector field $K$ we can choose a gauge such that the Lie derivative of the vector potentials $B_{\alpha}^{I}$ vanishes and choose adapted coordinates such that the isometry is just a translation $\left(\right.$ e.g. $\left.K=\frac{\partial}{\partial t}\right)$. All the fields $G_{\alpha \beta}, \bar{\phi}^{i}$ and $B_{\alpha}^{I}$ will then depend only on the remaining three coordinates $x^{a}(a=1,2,3)$ parametrizing the orbit space $\Sigma_{3}$ of the action of $K$. In these coordinates $K_{\alpha}$ has the form $K_{\alpha}=\left(\Delta k_{a}, \Delta\right)$ and the metric $g_{\alpha \beta}$ can be decomposed as

$$
g_{\alpha \beta}=\left(\begin{array}{cc}
-\frac{1}{\Delta} h_{a b}+\Delta k_{a} k_{b} & \Delta k_{b} \\
\Delta k_{a} & \Delta
\end{array}\right) .
$$

We will use the scaled metric $h_{a b}$ on the three dimensional orbit space $\Sigma_{3}$, since it leads to the standard form of the Lagrangian in 3 dimensions. In order to do all this we have to require $\Delta \neq 0 . h_{a b}$ is positive definite if $K$ is time-like, i.e. $\Delta>0$ and has the signature $(+--)$ if $K$ is space-like, i.e. $\Delta<0$. Similarly we decompose the vector fields $B_{\alpha}^{I}=\left(\widetilde{B}_{a}^{I}+k_{a} B^{I}, B^{I}\right)$ into pieces $\widetilde{B}_{a}^{I}$ and $B^{I}$ perpendicular and parallel to $K$. We can now rewrite the Lagrangian [compare Eq. (2.15)] in the form (apart from surface terms)

$$
\begin{aligned}
\widetilde{\mathscr{L}}= & \sqrt{h}\left(\frac{1}{2} R^{(3)}-\frac{1}{2} h^{a b}\left(\left\langle\bar{J}_{a}, \bar{J}_{b}\right\rangle-\frac{1}{\Delta} \partial_{a} B^{T} \mu(\bar{\phi}) \partial_{b} B+\frac{1}{2 \Delta^{2}} \partial_{a} \Delta \partial_{b} \Delta\right)+\frac{\Delta^{2}}{8} k_{a b} k^{a b}\right. \\
& \left.+\left(\widetilde{G}_{a b}+k_{a b} B\right)^{T}\left(-\frac{\Delta}{4} \mu(\bar{\phi})\left(\widetilde{G}^{a b}+k^{a b} B\right)+\frac{1}{2 \sqrt{h}} \varepsilon^{a b c} v(\bar{\phi}) \partial_{c} B\right)\right)
\end{aligned}
$$

where $R^{(3)}$ is the scalar curvature for $h_{a b}, k_{a b}=\partial_{a} k_{b}-\partial_{b} k_{a}, \widetilde{G}_{a b}=\partial_{a} \widetilde{B}_{b}-\partial_{b} \widetilde{B}_{a}$, and all indices are raised or lowered with the metric $h$. The Killing vector field is hypersurface orthogonal iff $k_{a b}=0$. 
If the original field configuration was a solution of the 4-dimensional field equations (2.16-18) then $h_{a b}, \Delta, k_{a}, B^{I}, \widetilde{B}_{a}^{I}$ and $\bar{\phi}^{i}$ are a solution of the three dimensional field equations derived from the action $\int_{\Sigma_{3}} \tilde{\mathscr{L}}$ and vice versa. The field equations for the 3 -dimensional vector fields $\widetilde{B}_{\mathrm{a}}^{\mathrm{I}}$ and $k_{a}$,

$$
\begin{gathered}
\nabla_{a}\left(-\Delta \mu(\bar{\phi})\left(\widetilde{G}^{a b}+k^{a b} B\right)+\frac{1}{\sqrt{h}} \varepsilon^{a b c} v(\bar{\phi}) \partial_{c} B\right)=0, \\
\nabla_{a}\left(\frac{\Delta^{2}}{2} k^{a b}+B^{T}\left(-\Delta \mu(\bar{\phi})\left(\widetilde{G}^{a b}+k^{a b} B\right)+\frac{1}{\sqrt{h}} \varepsilon^{a b c} v(\bar{\phi}) \partial_{c} B\right)\right)=0,
\end{gathered}
$$

can be considered as Bianchi identities for the dual potentials $C_{I}$ (which are just the time components $K^{\alpha} C_{\alpha I}$ of the dual potentials $C_{\alpha I}$ ) and $\tilde{\psi}$ (the twist potential).

Instead of using the definitions of $k_{a b}$ and $\widetilde{G}_{a b}$ we can treat them as independent fields and add Lagrange multipliers to the Lagrangian which guarantee that they are curls,

$$
\tilde{\mathscr{L}}^{\prime}=\tilde{\mathscr{L}}+\frac{1}{2} \varepsilon^{a b c}\left(C^{T}\left(\eta^{T}\right)^{-1} \partial_{a} \widetilde{G}_{b c}+\frac{1}{2}\left(\tilde{\psi}+C^{T}\left(\eta^{T}\right)^{-1} B\right) \partial_{a} k_{b c}\right) .
$$

The resulting field equations for $k_{a b}$ and $\widetilde{G}_{a b}$ are

$$
\begin{gathered}
k^{a b}=\frac{1}{\sqrt{h}} \varepsilon^{a b c} \frac{1}{\Delta^{2}} \omega_{c} \quad \text { with } \quad \omega_{a}=\partial_{a} \tilde{\psi}+\left(C^{T}\left(\eta^{T}\right)^{-1} \partial_{a} B-B^{T} \eta^{-1} \partial_{a} C\right), \\
\tilde{G}^{a b}+k^{a b} B=\frac{1}{\sqrt{h}} \varepsilon^{a b c} \frac{1}{\Delta} \mu^{-1}\left(v \partial_{c} B-\eta^{-1} \partial_{c} C\right) .
\end{gathered}
$$

Inserting these expressions into $\tilde{\mathscr{L}}^{\prime}$ we finally obtain the Lagrangian for the dimensionally reduced three dimensional theory,

$$
\begin{aligned}
\mathscr{L}^{(3)} & =\sqrt{h}\left(\frac{1}{2} R^{(3)}-\frac{1}{2} h^{a b}\left(\frac{1}{2 \Delta^{2}}\left(\partial_{a} \Delta \partial_{b} \Delta+\omega_{a} \omega_{b}\right)+\left\langle\bar{J}_{a}, \bar{J}_{b}\right\rangle-\frac{1}{\Delta} \partial_{a} A^{T} \hat{\bar{M}} \partial_{b} A\right)\right) \\
& \equiv \sqrt{h}\left(\frac{1}{2} R^{(3)}-\frac{1}{2} h^{a b} \partial_{a} \phi^{i} \partial_{b} \phi^{j} \gamma_{i j}(\phi)\right),
\end{aligned}
$$

and we can rewrite the twist vector in the explicitly $\bar{G}$-invariant form

$$
\omega_{a}=\partial_{a} \tilde{\psi}+A^{T} \bar{Y}^{-1} \partial_{a} A
$$

If all potentials $A$ for the vector fields vanish, then $\omega_{a}=\partial_{a} \tilde{\psi}$ and $(\Delta, \tilde{\psi})$ parametrize the well known $S L(2) / S O(2) \sigma$-model of pure gravity.

We have obtained a non-linear $\sigma$-model with a target space $\Phi$ parametrized by $\phi=(\Lambda, \tilde{\psi}, A, \bar{\phi})$ and coupled to (three dimensional) gravity. For a space-like Killing vector $(\Delta<0)$ the metric on $\Phi$ is positive definite, but for a time like-Killing vector ( $\Delta>0$, stationary solutions) the metric is indefinite with $2 k$ negative terms due to the fields $A$ originating from the $k$ vector fields in the 4-dimensional theory. 
From the Lagrangian (3.6) we obtain the field equations

$$
\begin{gathered}
R_{a b}^{(3)}-\partial_{a} \phi^{i} \partial_{b} \phi^{j} \gamma_{i j}(\phi)=0, \\
\nabla^{a}\left(\frac{\omega_{a}}{\Delta^{2}}\right)=0 \\
\nabla^{a}\left(\frac{\hat{\bar{M}}}{\Delta} \partial_{a} A+\bar{Y}^{-1} A \frac{\omega_{a}}{\Delta^{2}}\right)=0 \\
\nabla^{a}\left(\frac{\partial_{a} \Delta}{\Delta}-A^{T} \frac{\hat{\bar{M}}}{\Delta} \partial_{a} A+\tilde{\psi} \frac{\omega_{a}}{\Delta^{2}}\right)=0 \\
\nabla^{a}\left(\frac{1}{2} \bar{M}^{-1} \partial_{a} \bar{M}+\frac{1}{\hat{\bar{c}}}\left(A \partial_{a} A^{T} \frac{\hat{\bar{M}}}{\Delta}-\frac{1}{2} A \frac{\omega_{a}}{\Delta^{2}} A^{T} \bar{Y}^{-1}\right)_{\mathrm{pr}}\right)=0 .
\end{gathered}
$$

The metric on the space $\Phi$ and the field equation (3.8-9) are obviously invariant under

1. $\bar{G}$-transformations (acting on $\bar{\phi}$ and $A$ ),

2. "electromagnetic" gauge transformations (with a constant vector $a$ )

$$
\tilde{\psi} \rightarrow \tilde{\psi}+A^{T} \bar{Y}^{-1} a, \quad A \rightarrow A+a,
$$

3. twist gauge transformations

$$
\tilde{\psi} \rightarrow \tilde{\psi}+\chi
$$

4. scale transformations

$$
\Delta \rightarrow \zeta^{2} \Delta, \quad \tilde{\psi} \rightarrow \zeta^{2} \tilde{\psi}, \quad A \rightarrow \zeta A .
$$

On the submanifold $A=0$ there is the well known (infinitesimal) Ehlers transformation [38],

$$
\delta \Delta=2 \tilde{\psi} \Delta, \quad \delta \tilde{\psi}=\tilde{\psi}^{2}-\Delta^{2}, \quad \delta \bar{\phi}=0 .
$$

It may or may not be possible to extend this isometry to the whole manifold $\Phi$. We are interested in those cases where this is indeed possible and find that the metric on $\Phi$ is in addition invariant under

5. (infinitesimal) generalized Ehlers transformations

$$
\begin{gathered}
\delta \Delta=2 \tilde{\psi} \Delta, \quad \delta \tilde{\psi}=\tilde{\psi}^{2}-\Delta^{2}+A^{T} \hat{\bar{M}} A \Delta+O\left(A^{4}\right), \\
\delta \bar{\phi}=O\left(A^{2}\right), \quad \delta A=(\tilde{\psi}-\bar{Y} \hat{\bar{M}} \Delta) A+O\left(A^{3}\right),
\end{gathered}
$$

6. (infinitesimal) generalized Harrison transformations [20] (the commutator of the transformations $(3.10,14))$

$$
\begin{gathered}
\delta \Delta=O(A) \Delta, \quad \delta \tilde{\psi}=O(A), \\
\delta \bar{\phi}=O(A), \quad \delta A=(\tilde{\psi}-\bar{Y} \hat{\bar{M}} \Delta) a+O\left(A^{2}\right) .
\end{gathered}
$$

All these transformations will then form a non-compact Lie group $G$ and $\Phi$ is either the Riemannian symmetric space $G / H^{\prime}$, where $H^{\prime}$ is the maximal compact subgroup of $G(\Delta<0)$ or the pseudo-Riemannian symmetric space $G / H(\Delta>0)$, where $H$ is a non-compact real form of $H^{\prime}$. Moreover the submanifold where $A=0$ (i.e. the intersection $\left.H^{\prime \prime} / K=G / H \cap G / H^{\prime}\right)$ must be $S L(2) / S O(2) \times \bar{G} / \bar{H}$ and the 
generators of the coset space must transform under the two dimensional representation of $S L(2)$ in order to reproduce the scale transformations (3.12). In Appendix B we give a complete list of all combinations of groups $G, H, H^{\prime}, H^{\prime \prime}$ and $K$ which have these properties. For each of them we can start from the Lagrangian (3.6) for the three dimensional theory and reconstruct the Lagrangian (2.15) of the corresponding 4-dimensional theory.

For definiteness let us consider the stationary case with a pseudo-Riemannian symmetric space $G / H$, and let us assume that the group $G$ is simple. We proceed in a similar way as we did previously for $\bar{G}$. We choose a (possibly complex) irreducible matrix representation $\varrho$ of $G$ and a hermitian matrix $X$ satisfying $\varrho(\tau(g))$ $=X^{-1} \varrho\left(g^{-1}\right) X$, and define $\hat{P}=\varrho(P), \hat{M}=\hat{P}^{+} X \hat{P}=X \varrho(M)$. The Lagrangian (3.6) can then be rewritten in the form

$$
\begin{aligned}
\mathscr{L}^{(3)} & =\sqrt{h}\left(\frac{1}{2} R^{(3)}-\frac{1}{2}\left\langle J^{a}, J_{a}\right\rangle\right) \\
& \equiv \sqrt{h}\left(\frac{1}{2} R^{(3)}-\frac{\hat{c}}{8} h^{a b} \operatorname{Tr}\left(\hat{M}^{-1} \partial_{a} \hat{M} \hat{M}^{-1} \partial_{b} \hat{M}\right)\right),
\end{aligned}
$$

with a constant $\hat{c}$ depending on the representation $\varrho$ and on the particular $\sigma$-model. Introducing the currents [compare Eq. (A.11)]

$$
J_{a}=\frac{1}{2} M^{-1} \partial_{a} M,
$$

the field equations (3.8-9) can be rewritten in the form

$$
\begin{gathered}
R_{a b}^{(3)}=\left\langle J_{a}, J_{b}\right\rangle, \\
\nabla^{a} J_{a}=0 .
\end{gathered}
$$

As mentioned in Appendix A, not all of the conserved currents $J_{a}$ and not all of the field equations $\nabla J=0$ are independent.

In order to make the connection with the 4-dimensional equations of motion it is useful to observe that for any current $J^{a}$ defined on $\Sigma_{3}$ there is a corresponding current

$$
J^{\alpha}=\left(\Delta^{-1} J^{a}, 0\right)
$$

defined on the 4-dimensional space-time $\Sigma$ with vanishing Lie derivative with respect to $K$. Furthermore there is a related antisymmetric tensor field $J^{\alpha \beta} \equiv K^{\alpha} J^{\beta}$ $-J^{\alpha} K^{\beta}$ with the property

$$
J_{; \beta}^{\alpha \beta}=K^{\alpha} J_{; \beta}^{\beta}=\Delta^{-1} K^{\alpha} J_{; b}^{b} .
$$

We can thus convert conservation laws on $\Sigma_{3}$ into Maxwell-like equations on $\Sigma$. In some cases this gives the possibility of avoiding difficulties with the vanishing of $\Delta$ (compare the proof of Theorem 2.8).

Later on in Chap. 5 we will study solutions with asymptotically Euclidean $\Sigma_{3}$ and $\sigma$-model fields which have an asymptotic multipole expansion,

$$
M(x) \sim \sum_{n=0}^{\infty} r^{-n} M_{n}(\vartheta, \varphi) .
$$


Without loss of generality we can assume that they have the asymptotic values $\Delta_{0}=1, \tilde{\psi}_{0}=A_{0}=0, \bar{M}_{0}=\mathbf{1}$ corresponding to $P_{0}=\mathbf{1}, M_{0}=\mathbf{1}$ or $\hat{M}_{0}=X$. The charges $\lim _{r \rightarrow \infty} r J_{a} x^{a}=-\frac{1}{2} M_{0}^{-1} M_{1}$ have values in the Lie algebra of $G$, but in fact those in the Lie algebra of $H$ vanish identically. The subgroup $H \subset G$ leaves the asymptotic value $P_{0}$ invariant and transforms the charges according to

$$
H \ni h: X^{-1} M_{1} \rightarrow h X^{-1} M_{1} h^{-1} \equiv X^{-1}\left(h^{+}\right)^{-1} M_{1} h^{-1} .
$$

The precise form of this action of $H$ on the charges depends on the particular $\sigma$-model under consideration. There are, however, in all cases, generators of $H$ which differ from the transformations $(3.14,15)$ by suitable multiples of the transformations (3.10-12). The names generalized Ehlers and Harrison transformation shall henceforth refer to these linear combinations and we can use them to transform the (electric and magnetic) vector charges and the NUT charge into the mass and the scalar charges and vice versa (compare Eqs. (3.10-15)).

Remark (Static Truncation). If we apply the staticity constraints to the $\sigma$-model described by the Lagrangian (3.6) we obtain a consistent truncation, the corresponding "static" $\sigma$-model.

Appendix B contains some examples of such static $\sigma$-models. At this point we just want to mention the static truncation $S O(n, 1) / S O(n-1,1)$ of the generalized Einstein-Maxwell case $S U(n, 1) / S(U(n-1,1) \times U(1))$. The special case $n=2$ corresponds to the Einstein-Maxwell theory.

\subsection{Dimensional Reduction from 3 to 2 Dimensions}

Stationary, axially symmetric configurations of the 4-dimensional theory are characterized by their invariance under two commuting Killing vector fields $K$ respectively $K^{\prime}$ describing time-translations respectively axial rotations. Similar to the case of one Killing vector we can perform a "dimensional reduction" from 4 to 2 dimensions. Since we want to make use of the $\sigma$-model structure obtained for the 3-dimensional theory after suitable dualizations we prefer to employ a two step procedure. First we use one Killing vector field to reduce from 4 to 3 dimensions, and then the second one to do the step to 2 dimensions. Depending on whether we take the Killing vector field $K=\frac{\partial}{\partial t}$ or $K^{\prime}=\frac{\partial}{\partial \varphi}$ to perform the reduction from 4 to 3 dimensions we end up with two different $\sigma$-models corresponding to $G / H$ respectively $G / H^{\prime}$, parametrized by the matrices $M$ respectively $M^{\prime}$.

In order to be able to do the step from 3 to 2 dimensions we first have to make sure that the Lie derivative of the 3-dimensional fields $h_{a b}$ and $M$ with respect to the second Killing vector vanishes. For $h_{a b}$ this is a direct consequence of the commutativity of the two Killing vectors. For the electromagnetic potentials $A$ we find that their Lie derivative has to be constant. This constant has to vanish for asymptotically trivial configurations with a regular rotation axis. In adapted coordinates the vanishing Lie derivative of $h_{a b}$ and $M$ means they are independent of $t$ and $\varphi$. Hence the only non-trivial step in the reduction from 3 to 2 dimensions concerns the decomposition of the 3 -metric $h_{a b}$ which we parametrize in analogy to 
Eq. (3.1) in the form

$$
h_{a b}=\left(\begin{array}{cc}
\lambda^{2} \tilde{h}_{k l}+\varrho^{2} b_{k} b_{l} & \varrho^{2} b_{l} \\
\varrho^{2} b_{k} & \varrho^{2}
\end{array}\right) .
$$

In terms of these new fields the Lagrangian (3.6) becomes

$$
\mathscr{L}^{(2)}=\varrho \sqrt{\tilde{h}}\left[-\frac{1}{2} \tilde{R}-\frac{\varrho^{2}}{8 \lambda^{2}} b_{k l} b^{k l}+\frac{1}{8}\left\langle M^{-1} \partial M, M^{-1} \partial M\right\rangle-\lambda^{-1} \partial \lambda \varrho^{-1} \partial \varrho\right] .
$$

A novel feature for $d=2$ is that $\lambda$ cannot be used to remove the factor $\varrho$ multiplying $\sqrt{\tilde{h}}$ in front of the Lagrangian. This is a consequence of the conformal invariance of the 2-dimensional theory.

A simplification occurs with the vector fields $b_{k}$ which lose their dynamical degrees of freedom in 2 dimensions. From the field equation

$$
\nabla_{k}\left(\varrho^{3} \lambda^{-2} b^{k l}\right)=0,
$$

it follows that the dual field strength $* b=\frac{1}{2 \sqrt{h}} \varepsilon^{k l} \varrho^{3} \lambda^{-2} b_{k l}$ obeys the equation

$$
\varepsilon^{k l} \partial_{l}^{*} b=0 \text {, }
$$

and hence ${ }^{*} b=$ const. For asymptotically Minkowskian solutions $* b$ vanishes at infinity, and hence the constant vanishes, and consequently $b_{k l}=0$, i.e. the Killing vector fields are hypersurface orthogonal. This is our version of the "Generalized Papapetrou Theorem" [13]. Then $\mathscr{L}$ simplifies to

$$
\mathscr{L}^{(2)}=\varrho \sqrt{\tilde{h}}\left[-\frac{1}{2} \widetilde{R}+\frac{1}{8}\left\langle M^{-1} \partial M, M^{-1} \partial M\right\rangle-\lambda^{-1} \partial \lambda \varrho^{-1} \partial \varrho\right] .
$$

The field equations derived from $\mathscr{L}^{(2)}$ are (we omit the field equation for $\lambda$, since it is of no relevance)

$$
\begin{gathered}
\tilde{R}_{k l}-\frac{1}{2} \tilde{h}_{k l} \tilde{R}=\frac{1}{4}\left\langle M^{-1} \partial_{k} M, M^{-1} \partial_{l} M\right\rangle-2 \lambda^{-1} \partial_{(k} \lambda \varrho^{-1} \partial_{l)} \varrho \\
-\frac{1}{2} \tilde{h}_{k l}\left(\frac{1}{4}\left\langle M^{-1} \partial M, M^{-1} \partial M\right\rangle-2 \lambda^{-1} \partial \lambda \varrho^{-1} \partial \varrho\right), \\
\nabla\left(\varrho M^{-1} \partial M\right)=0, \\
\nabla \partial \varrho=0 .
\end{gathered}
$$

The 2-dimensional metric $\tilde{h}_{k l}$ can be brought to the conformally flat form $\tilde{h}_{k l}=\tilde{h} \delta_{k l}$ by a suitable choice of coordinates. Finally $\tilde{h}$ can be absorbed into the conformal factor $\lambda$ leading to $\tilde{h}_{k l}=\delta_{k l}$. Since the field equations for $M$ and $\varrho$ are conformally invariant they are independent of $\lambda$ and hence the same as in flat space. In particular $\varrho$ is a harmonic function on $\mathbf{R}^{2}$. Together with its conjugate harmonic function $z$ defined by $\partial z=-* \partial \varrho$ (note that $* * \partial=-\partial$ in a 2 dimensional space with definite metric) it provides a canonical coordinatization of the 2-dimensional reduced manifold as long as $\partial \varrho \neq 0$ (Weyl's canonical coordinates). With this choice, which we shall always make from now on, the equation for $M$ is that of a generalized (not translationally invariant) non-linear $\sigma$-model on flat space independent of $\lambda$. 
Equation (3.29) turns into an equation for $\lambda$, since the left-hand side vanishes for $\tilde{h}_{k l}=\delta_{k l}$. One finds

$$
\begin{gathered}
\lambda^{-1} \partial_{z} \lambda=\frac{\varrho}{4}\left\langle M^{-1} \partial_{\varrho} M, M^{-1} \partial_{z} M\right\rangle, \\
\lambda^{-1} \partial_{\varrho} \lambda=\frac{\varrho}{8}\left(\left\langle M^{-1} \partial_{\varrho} M, M^{-1} \partial_{\varrho} M\right\rangle-\left\langle M^{-1} \partial_{z} M, M^{-1} \partial_{z} M\right\rangle\right) .
\end{gathered}
$$

From these equations $\lambda$ can be computed by a simple integration once $M$ is known. The integrability conditions are satisfied if Eq. (3.30) is fulfilled.

\section{Spherically Symmetric Solutions}

The system of Eqs. (2.2-3) looks deceptively simple due to its elegant mathematical description. But it has to be remembered that it describes rather complex and complicated physical situations and mathematical structures. Most of its explicitly known solutions are therefore distinguished by some symmetry properties of the remaining 3-dimensional Riemannian space $\Sigma_{3}$ and the $\sigma$-model fields $\phi^{i}(x)$ reducing the number of essential variables. The maximal symmetry group for $\Sigma_{3}$ is the 6-parameter euclidean group of motions, which singles out the trivial "vacuum" solution, 4-dimensional Minkowski space with vanishing vector field strengths and constant scalar fields. A physically more interesting class of solutions are the spherically symmetric solutions with an isometry group $S O(3)$ acting on 2-dimensional orbits. Note that if the NUT-charge is non-zero the action of $S O(3)$ on the 4-dimensional space-time has 3-dimensional orbits. Using polar coordinates the metric of $\Sigma_{3}$ can be parametrized as $d s^{2}=h_{a b} d x^{a} d x^{b}=d r^{2}+f(r)^{2}\left(d \vartheta^{2}\right.$ $\left.+\sin ^{2} \vartheta d \varphi^{2}\right)$. The Eqs. (2.2-3) become under these circumstances

$$
\begin{gathered}
f^{-2} \frac{d}{d r}\left(f^{2} \frac{d \phi^{i}}{d r}\right)+\Gamma_{j k}^{i}(\phi) \frac{d \phi^{j}}{d r} \frac{d \phi^{k}}{d r}=0, \\
R_{r r} \equiv-2 f^{-1} \frac{d^{2} f}{d r^{2}}=\gamma_{i j}(\phi) \frac{d \phi^{i}}{d r} \frac{d \phi^{j}}{d r}, \\
R_{\varphi \varphi}=R_{\vartheta \vartheta} \equiv f^{-2}\left(\frac{d}{d r} f \frac{d f}{d r}-1\right)=0 .
\end{gathered}
$$

The last equation has the general solution

$$
f(r)^{2}=\left(r-r_{0}\right)^{2}+c .
$$

Introducing $\tau(r) \equiv-\int_{r}^{\infty} f^{-2}(s) d s$, which is a harmonic function on $\Sigma_{3}$ equipped with the metric $h_{a b}$, Eq. (4.1a) becomes

$$
\frac{d^{2} \hat{\phi}^{i}}{d \tau^{2}}+\Gamma_{j k}^{i}(\phi) \frac{d \hat{\phi}^{j}}{d \tau} \frac{d \hat{\phi}^{k}}{d \tau}=0
$$

with $\phi^{i}(r)=\hat{\phi}^{i}(\tau(r))$. This is the equation for a geodesic in the symmetric space $G / H$. The decomposition of $\phi: \Sigma_{3} \rightarrow G / H$ into a harmonic map $\tau: \Sigma_{3} \rightarrow \mathbf{R}^{1}$ and a geodesic 
$\hat{\phi}: \mathbf{R}^{1} \rightarrow G / H$ illustrates a general theorem on harmonic maps [39] according to which the composition of a harmonic map with a totally geodesic one is again harmonic. Since $\hat{\phi}$ describes a geodesic we get

$$
\gamma_{i j}(\hat{\phi}) \frac{d \hat{\phi}^{i}}{d \tau} \frac{d \hat{\phi}^{j}}{d \tau}=2 v^{2}=\text { const } .
$$

From (4.1) we find $c=-v^{2}$. It is however important to notice that $v^{2}$ can in general have either sign due to the indefiniteness of the metric of $G / H$. The behaviour of the metric function $f^{2}$ and correspondingly of the affine parameter $\tau(r)$ depends crucially on the sign of $v^{2}$. If $v^{2}>0$ the function $f^{2}$ has two simple zeros at $r=r_{0} \pm v$ $\left(v \equiv \sqrt{v^{2}}\right)$, causing $\tau(r)=\frac{1}{2 v} \ln \left(\frac{r-r_{0}-v}{r-r_{0}+v}\right)$ to tend to $-\infty$ at $r=r_{0}+v$. Hence the geodesic in $G / H$ starts off at $\tau=0$ from some value $\phi_{0}$ - the asymptotic value of $\phi$ at $r=\infty$ in $\Sigma_{3}$-and approaches for infinite affine parameter some other value $\phi_{\mathscr{H}}$, the value of $\phi$ at $r=r_{0}+v$, the position of the horizon for black hole solutions. For $v^{2}=0$ the two zeros of $f^{2}$ merge and $\tau(r)$ becomes equal to $\tau(r)=-\left(r-r_{0}\right)^{-1}$. The behaviour of the geodesics is similar as for $v^{2}>0$. From Eq. (4.1) it follows that in this case $R_{a b}=0$, and hence $h_{a b}=\delta_{a b}$, i.e. $\Sigma_{3}$ is flat. On the other hand if $v^{2}<0$, i.e. the geodesic is "space-like", $f^{2}$ is always positive and the affine parameter $\tau(r)$ $=v^{-1}\left(\arctan \frac{r-r_{0}}{v}-\frac{\pi}{2}\right)$ runs from $\tau=0$ at $r=\infty$ to $\tau=-\frac{\pi}{v}$ at $r=-\infty$. Hence the "space-like" geodesics have finite length.

In order to illustrate this typical behaviour of the geodesics we take the simplest example allowing both signs of $v^{2}$. Since pure gravity leads to $G / H$ $=S L(2) / S O(2)$, which has positive definite metric, we choose the Einstein-Maxwell theory with $G / H=S U(2,1) / S(U(1,1) \times U(1)$ with the signature $(++--)$. For spherically symmetric solutions we can restrict ourselves to the subspace of strongly static solutions corresponding to $(G / H)_{\mathrm{st}}=S O(2,1) / S O(1,1)$, since a possible magnetic field can be trivially eliminated by a duality transformation. The corresponding line element of $\Phi$ is

$$
d s^{2}=\frac{d \Delta^{2}}{2 \Delta^{2}}-\frac{2 d A^{2}}{\Delta}
$$

where $\Delta$ respectively $A$ are the gravitational respectively electric potential. The geodesic equations are

$$
\ddot{\Delta}-\Delta^{-1} \dot{\Delta}^{2}-2 \dot{A}^{2}=0, \quad \ddot{A}-\Delta^{-1} \dot{\Delta} \dot{A}=0 .
$$

They can easily be solved directly with the boundary conditions $\Delta(0)=1, A(0)=0$ according to the asymptotic behaviour at $r=\infty$. One obtains the well-known Reissner-Nordstrøm solutions. For $v^{2}>0$ one finds

$$
\Delta=\frac{\sinh ^{2} \beta}{\sinh ^{2}(\beta-v \tau)}, \quad A=\frac{\sinh v \tau}{\sinh (\beta-v \tau)},
$$

where $m=v \operatorname{coth} \beta$ and $q=v / \sinh \beta$ are the mass and the charge of the solution. From Eq. (4.5) one obtains the relation $m^{2}-q^{2}=v^{2}$. 
The equation

$$
\Delta=(A+\cosh \beta)^{2}-\sinh ^{2} \beta
$$

shows that the geodesics are parabolae hitting the axis $\Delta=0$ at $A=\sinh \beta-\cosh \beta$ $=-q^{-1}\left(m-\sqrt{m^{2}-q^{2}}\right)$ for $\tau=-\infty$ respectively $r=r_{0}+\sqrt{m^{2}-q^{2}}=m+\sqrt{m^{2}-q^{2}}$ for the standard choice $r_{0}=m$. This is the position of the event horizon. The solution for $v^{2}=0$ is obtained letting $v, \beta \rightarrow 0$ with their ratio tending to $m$. The result is the extreme Reissner-Nordstrøm solution $\left(q^{2}=m^{2}\right)$ given by

$$
\Delta=(1-m \tau)^{-2}, \quad A=m \tau(1-m \tau)^{-1} .
$$

This time the parabola $\Delta=(A+1)^{2}$ just touches the $\Delta=0$ axis at $A=-1$ for $r=r_{0}$, the position of the degenerate horizon.

The space-like geodesics with $v^{2}<0$ are obtained by analytic continuation from $v^{2}>0$ replacing $v$ by $i v$ and $\beta$ by $i \beta$. One obtains the over-extreme solutions given by $\left(v=\sqrt{\left|v^{2}\right|}\right)$

$$
\Delta=\frac{\sin ^{2} \beta}{\sin ^{2}(\beta-v \tau)}, \quad A=\frac{\sin v \tau}{\sin (\beta-v \tau)} .
$$

These geodesics do not reach $\Delta=0$, but $\Delta$ tends to infinity for the finite value $\tau=\frac{\beta-\pi}{v}$ of the affine parameter, corresponding to $r=r_{0}-v \operatorname{coth} \beta=r_{0}-m$. This is the position of the naked singularity.

Since the metric (4.5) is that of 2-dimensional de Sitter space we may illustrate these facts by means of a Carter-Penrose diagram shown in Fig. 1. This figure may be obtained by setting $\lambda^{-1}=\Delta$ and $\tau=2 A$ in Fig. 2.2b of [13, p. 66]. In Fig. 1 light rays are at $45^{\circ}$ and the dashed (dots) indicate curves of constant $\Delta(A)$ respectively. The vanishing of $\Delta$ corresponds to future or past time-like infinity. If $v^{2} \geqq 0$ the

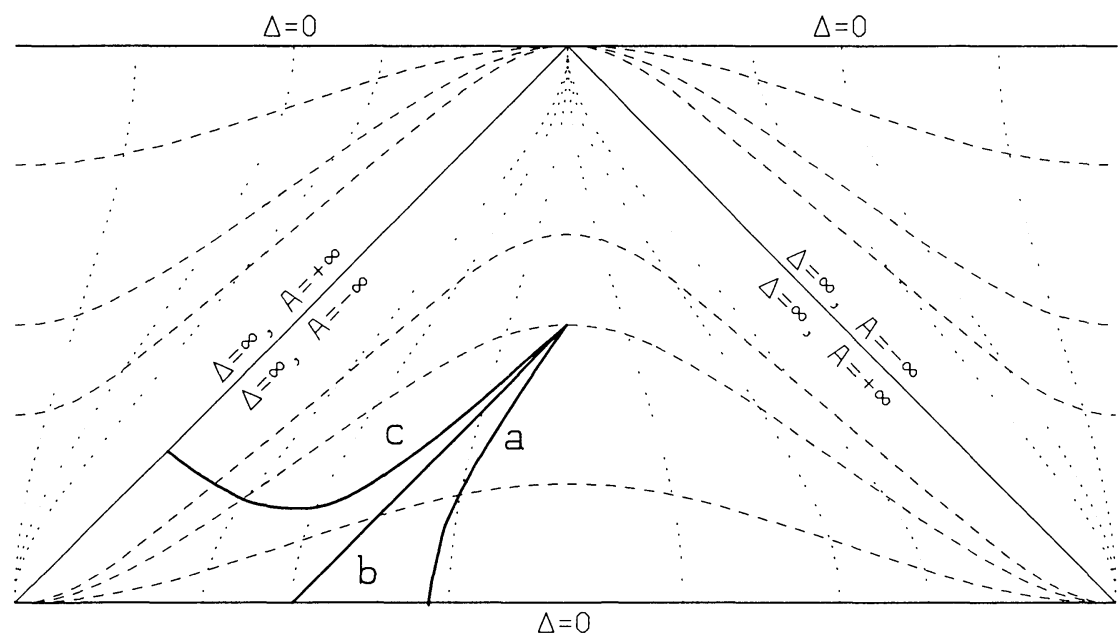

Fig. 1. Carter-Penrose diagram for 2-dimensional de Sitter space. The curves $a, b, c$ are examples of time-like, light-like, space-like geodesic segments respectively corresponding to the solutions $(4.7-10)$ 
geodesics start from an interior point at $\tau=0$ and reach past time-like infinity at $\tau=-\infty$. The space-like geodesics, $v^{2}<0$, never reach infinity but intersect the lines $\Delta=\infty$ where the Killing vector $\frac{\partial}{\partial A}$ becomes light-like.

Summarizing we arrive at the following picture. Each of the geodesics corresponding to a solution with the required asymptotic behaviour has to pass through $\phi_{0}=(\Delta(0), A(0))=(1,0)$ for $\tau=0$ and is uniquely determined by its tangent vector $\dot{\phi}(0)$ there. $\dot{\phi}(0)=(\dot{\Delta}(0), \dot{A}(0))=(m, q)$ is given in terms of the "global charges" of the conserved currents $J_{a}$ of Eq. (3.17). The group $S O(1,1)$ leaves $\phi_{0}$ fixed and acts transitively on the tangent vectors of fixed length $v^{2}$. Therefore it is possible to get the charged solution Eq. (4.7) from the Schwarzschild solution with the same value of $v^{2}$ through a Harrison transformation.

In the general case we obtain the following equations for the geodesics describing spherically symmetric black holes:

$$
\begin{gathered}
\frac{d}{d \tau}\left(\Delta^{-1} \hat{\bar{M}} \frac{d A}{d \tau}\right)=0 \\
\frac{d}{d \tau}\left(\Delta^{-1} \frac{d \Delta}{d \tau}-\Delta^{-1} A^{T} \hat{\bar{M}} \frac{d A}{d \tau}\right)=0 \\
\frac{d}{d \tau}\left(\frac{1}{2} \bar{M}^{-1} \frac{d \bar{M}}{d \tau}+\Delta^{-1} \frac{1}{\hat{\bar{c}}}\left(A \frac{d A^{T}}{d \tau} \hat{\bar{M}}\right)_{\mathrm{pr}}\right)=0 .
\end{gathered}
$$

Remark. In distinction to the case of pure gravity the assumption of spherical symmetry and the absence of a NUT charge does not mean that the solutions have to be strongly static in the sense defined in Sect. 2.2

From the asymptotic behaviour of the geodesics we learn how to fix the arbitrary constants obtained by integrating these equations:

$$
\begin{gathered}
\Delta^{-1} \hat{\bar{M}} \frac{d A}{d \tau}=-Q_{A}, \\
\Delta^{-1} \frac{d \Delta}{d \tau}-\Delta^{-1} A^{T} \hat{\bar{M}} \frac{d A}{d \tau}=2 m, \\
\frac{1}{2} \bar{M}^{-1} \frac{d \bar{M}}{d \tau}+\Delta^{-1} \frac{1}{\hat{\bar{c}}}\left(A \frac{d A^{T}}{d \tau} \hat{\bar{M}}\right)_{\mathrm{pr}}=\bar{Q} .
\end{gathered}
$$

From Eq. (4.4) we get

$$
\frac{1}{2}\left(\Delta^{-1} \frac{d \Delta}{d \tau}\right)^{2}+\frac{1}{4}\left\langle\bar{M}^{-1} \frac{d \bar{M}}{d \tau}, \bar{M}^{-1} \frac{d \bar{M}}{d \tau}\right\rangle-\Delta^{-1} \frac{d A^{T}}{d \tau} \hat{\bar{M}} \frac{d A}{d \tau}=2 v^{2}
$$

Putting $\tau=0$ in this equation we obtain the relation

$$
m^{2}+\frac{1}{2}\langle\bar{Q}, \bar{Q}\rangle-\frac{1}{2} Q_{A}^{T} Q_{A}=v^{2} .
$$

Although it seems quite impossible to solve Eqs. (4.12) in closed form in the general case, it is possible to study the behaviour of the geodesics qualitatively. We are particularly interested in the asymptotic behaviour when $\tau \rightarrow-\infty$, where we 
approach the horizon. We have to anticipate the boundary conditions on the horizon, which will be discussed in the following section:

$$
\Delta(-\infty)=0, \quad \bar{M}(-\infty)=\bar{M}_{\mathscr{H}}<\infty, \quad A(-\infty)=A_{\mathscr{H}}<\infty .
$$

These boundary conditions imply in particular that $\frac{d \bar{M}}{d \tau}$ must vanish in the limit $\tau \rightarrow-\infty$, and we obtain the following relations between the charges and boundary values at the horizon:

$$
2 m-A_{\mathscr{H}}^{T} Q_{A}=\lim _{\tau \rightarrow-\infty} \frac{d \ln \Delta}{d \tau}, \quad \bar{Q}=-\frac{1}{\hat{\bar{c}}}\left(A_{\mathscr{H}} Q_{A}^{T}\right)_{\mathrm{pr}} .
$$

There are two essentially different situations:

1. $\frac{d \ln \Delta}{d \tau} \underset{\tau \rightarrow-\infty}{\rightarrow} \lambda>0$. This is only possible for $v^{2}>0$, because the negative contribution in Eq. (4.13) vanishes for $\Delta \rightarrow 0$. Obviously $\Delta$ behaves like

$$
\Delta \sim e^{\lambda \tau} \text { for } \tau \rightarrow-\infty \text {. }
$$

Considering $A$ and $\bar{M}$ as functions of $\Delta$ one finds $A=A_{\mathscr{H}}+O(\Delta)$ and $\bar{M}=\bar{M}_{\mathscr{H}}$ $+O(\Delta)$. The quantity $2 \pi \lambda$ is the surface gravity $\kappa$ times the area of the horizon.

2. $\frac{d \ln \Delta}{d \tau} \underset{\tau \rightarrow-\infty}{\rightarrow} 0$ describes solutions with a degenerate horizon. Analyzing Eqs. (4.11) together with the boundary conditions (4.15) one sees that this is only possible for $v^{2}=0$. The asymptotic behaviour is, in this case,

$$
\Delta \sim \frac{2}{Q_{A}^{T} \hat{\bar{M}}_{\mathscr{H}}^{-1} Q_{A}} \tau^{-2} \text { for } \tau \rightarrow-\infty
$$

with $A=A_{\mathscr{H}}+O(\sqrt{\Delta})$ and $\bar{M}=\bar{M}_{\mathscr{H}}+O(\Delta)$. In addition to the relations (4.16) there is a series of constraints on $\bar{M}$. The coefficients $C_{k}$ of the expansion $\bar{M}=\bar{M}_{\mathscr{H}} \exp \sum_{k=1}^{\infty} C_{k} \Delta^{k}$ must satisfy the equations

$$
k\left(k+\frac{1}{2}\right)\left(Q_{A}^{T} \hat{\bar{M}}_{\mathscr{H}}^{-1} Q_{A}\right) C_{k}-\frac{1}{\hat{\bar{c}}}\left(\hat{C}_{k} \hat{\bar{M}}_{\mathscr{H}}^{-1} Q_{A} Q_{A}^{T}\right)_{\mathrm{pr}}=\mathrm{rhs}, \quad k=1,2, \ldots,
$$

where the rhs depends only on $C_{l}$ with $l<k$ and vanishes for $k=1$. This is an inhomogeneous linear system for the $C_{k}$. For $k$ large enough there will be no solutions of the homogeneous system and we can solve the equations recursively. For small $k$ (and in particular for $k=1$ ) we must, however, look for solutions of the homogeneous system. A very simple example of this situation is provided by the extremal solution of [40] for the $\sigma$-model with $G / H=S L(3) / S O(2,1)$ and $\bar{G} / \bar{H}$ $=G L(1)$. The constraints require in this case that the value of the scalar field $\lambda_{22}$ on the horizon is $(Q / P)^{2 / 3}$, where $Q$ is the electric charge and $P$ the magnetic charge, i.e. neither $Q$ nor $P$ are allowed to vanish (compare also [41]).

The results of this chapter can be summarized in the following

Proposition 4.1. Every stationary spherically symmetric black hole solution is characterized by a time-like or light-like geodesic segment in $G / H$ starting at the 
point $M_{0}$ (corresponding to $r=\infty$ ) and running to the boundary $\Delta=0$. In general not every time-like geodesic leads to a black hole solution.

Remark. In Sect. 6 we will show that all such "time-like" black holes are Harrisontransformed Schwarzschild solutions (compare Theorem 6.3). Hence the scalar charges cannot be chosen freely but are determined by the other charges.

\section{Boundary Conditions for Black Holes}

In this section we shall consider the boundary conditions for our solutions. From our Generalized Serini-Einstein-Pauli-Lichnerowicz Theorem 2.8 we expect that non-trivial solutions must have singularities and/or more than one infinite region. Thus we look for solutions with event horizons hiding these singularities and/or infinite regions. Just as in our treatment of Theorem 2.8 we shall need for our arguments the existence of globally defined potentials. Therefore we shall assume that the exterior region of the black holes is simply connected. Furthermore most of our arguments would require a special treatment for the degenerate case. Thus in what follows we shall assume unless otherwise stated that all horizons are nondegenerate. We now discuss the boundary conditions at infinity and at the horizon.

We begin with infinity. The boundary conditions at infinity can be discussed in terms of the 3-dimensional space $\Sigma_{3}$ introduced in Sect. 3. For the EinsteinMaxwell theory this question has been thoroughly discussed by Simon [33]. As mentioned by Simon his results can be extended to a larger class of theories including those considered here. Accordingly we require the following asymptotic behaviour (holding in a suitable coordinate system) of the fields defined on $\Sigma_{3}$ :

$$
\begin{gathered}
h_{a b}=\delta_{a b}+O\left(\frac{1}{r}\right), \\
M=M_{0}+\frac{1}{r} M_{1}+O\left(\frac{1}{r^{2}}\right),
\end{gathered}
$$

where $M_{0}$, corresponding to the "vacuum" solution, is conveniently chosen as the unit element 1 of $G$. The behviour of the individual fields (after a suitable choice of gauge) in $M$ is

$$
\begin{gathered}
\Delta=1-\frac{2 m}{r}+O\left(\frac{1}{r^{2}}\right), \quad \tilde{\psi}=\frac{n}{r}+O\left(\frac{1}{r^{2}}\right), \\
A=\frac{Q_{A}}{r}+O\left(\frac{1}{r^{2}}\right), \quad \bar{M}=\mathbf{1}-\frac{2 \bar{Q}}{r}+O\left(\frac{1}{r^{2}}\right),
\end{gathered}
$$

where $m$ is the total mass, $n$ is the NUT-charge, $Q_{A}$ a vector of "electric" respectively "magnetic" charges and $\bar{Q}$ a hermitian matrix of scalar charges. In order to have asymptotically Minkowskian geometry the NUT-charge has to vanish, i.e. we require $n=0$.

We now turn to the event horizon which can be rotating or non-rotating.

Definition 5.1. A non-rotating event horizon is one whose null generator $l^{\alpha}$ coincides with the Killing vector field $K^{\alpha}$ on the horizon. 
Hawking's strong rigidity theorem [34] shows that in vacuum a rotating black hole must posess an additional Killing vector, i.e. must be axisymmetric. It seems plausible that this theorem can be generalized to Einstein-Maxwell theory and to the theories we are considering in this paper. However in view of the counterexamples quoted in Sect. 2.2 there is room for some caution about whether the matter fields need necessarily be axisymmetric. In this paper we shall assume that a rotating black hole is axisymmetric and that it is causal, i.e. that the axial Killing vector $K^{\prime}$ is space-like on and outside the horizon, apart from the rotation axis where it vanishes. We shall discuss single stationary axisymmetric black holes in Sect. 7.

For non-rotating horizons the exterior domain is either static or not. The static case is treated in Sects. 4 and 6.

Hawking [34] showed for the vacuum case that strict stationarity of the exterior domain implies staticity. The generalization of this result to electromagnetism has not yet been achieved (see [42] for a recent discussion which corrects a contrary claim in [13]). In any case even if Hawking's result could be generalized to include electromagnetism or the more general fields discussed in tis article it would still leave open the possibility of non-rotating holes with ergoregions. Our generalized Lichnerowicz Theorem renders this possibility less plausible than one might otherwise think, but there remains a substantial gap in the rigorous chain of uniqueness arguments, since it does not seem possible to treat this case with present day techniques.

In summary if the strong rigidity theorem can be generalized and anyhow in all the cases considered in this paper the event horizon is a Killing horizon in the sense of Carter [13].

Regardless of whether the horizon is a Killing horizon or not it follows that on the horizon

$$
T_{\alpha \beta} l^{\alpha} l^{\beta}=0
$$

This implies that

1. The scalar fields are constant along the null generators

$$
l^{\alpha} \partial_{\alpha} \bar{\phi}^{i}=0
$$

2. The electric and magnetic fields satisfy

$$
l^{\gamma} F_{\gamma[\alpha} l_{\beta]}=0, \quad l^{\gamma *} F_{\gamma[\alpha} l_{\beta]}=0 .
$$

If $l$ coincides on the horizon with a Killing vector field $L$ then the surface gravity $\kappa$ defined by

$$
\kappa=\sqrt{\frac{1}{2} L_{\alpha ; \beta} L^{\beta ; \alpha}}
$$

is constant on each connected component of the horizon. If one further assumes that the fields have vanishing Lie derivative with respect to $L$ one finds that the vector fields $L^{\beta *} F_{\alpha \beta}$ are curlfree. We can thus introduce (at least locally) potentials $\Psi$ by

$$
L^{\beta *} F_{\alpha \beta}=\partial_{\alpha} \Psi
$$


These potentials are constant on each connected component of the horizon. Note that unlike $\Psi$ the scalar fields $\bar{\phi}$ have to be finite but not necessarily constant on the horizon.

Let us now consider the strictly stationary case which includes in particular the static case. The horizon occurs at the boundary of $\Sigma_{3}$ where $\Delta$ vanishes. The surface gravity of the horizon is given by

$$
\kappa^{2}=\frac{1}{4} h^{a b} \partial_{a} \Delta \partial_{b} \Delta
$$

which must be constant on each connected component of the horizon, as must the potentials $A$. Moreover, being orthogonal to the horizon, $K$ satisfies there the hypersurface orthogonality condition $K_{[\alpha ; \beta} K_{\gamma]}=0$. This implies that $\omega_{a}$ vanishes and $\tilde{\psi}$ is constant on each connected component of the horizon. In addition the geometry determined by the physical 3-metric $\Delta^{-1} h_{a b}$ should remain regular as one approaches the boundary $\Delta \downarrow 0$. If $\kappa \neq 0$, i.e. for a non-degenerate horizon, this implies that $\Delta$ vanishes as the geodesic distance (in the scaled 3-metric $h_{a b}$ ) from the boundary and that (in suitable coordinates) the same holds true for the induced 2-metric on the surfaces $\Delta=$ const. In terms of the physical 3-metric the boundary is a totally geodesic 2-surface and each connected component has the topology of a 2 -sphere. The reader may check that these conditions are satisfied by the spherical metric $h_{a b}$ used in Sect. 4.

The boundary conditions in the stationary axisymmetric case are more complicated because one must consider the axis as well as the horizon. However they are essentially the same as those discussed by Carter [13] in the EinsteinMaxwell case. For one or several black holes the argument he gives to prove the existence of the global coordinates $\varrho$ and $z$ goes through in our case. In these coordinates the 2-dimensional space is the half-plane $\Sigma_{2}=\{(z, \varrho): \varrho \geqq 0\}$. Both the horizon and (the exterior part of) the rotation axis are represented by the $z$-axis $(\varrho=0)$. Each connected component of the horizon corresponds to a segment of the $z$-axis with $\Delta^{\prime}>0$ whereas $\Delta^{\prime}=0$ on the rotation axis.

The boundary conditions at infinity $\left(r=\sqrt{z^{2}+\varrho^{2}} \rightarrow \infty\right)$ are now [compare Eqs. $(5.1,2)]$

$$
\lambda=1+O\left(\frac{1}{r}\right), \quad M=M_{0}+\frac{1}{r} M_{1}+O\left(\frac{1}{r^{2}}\right) .
$$

The corresponding behaviour of $M^{\prime}$ is more complicated due to the non-trivial asymptotic behaviour of the axial Killing vector field and involves the angular momentum. For vanishing NUT-charge one gets

$$
\begin{gathered}
\Delta^{\prime}=\varrho^{2}\left(1+\frac{2 m}{r}+O\left(\frac{1}{r^{2}}\right)\right), \quad \tilde{\psi}^{\prime}=\frac{2 J\left(3 \varrho^{2} z+2 z^{3}\right)}{r^{3}}+O\left(\frac{1}{r}\right), \\
A^{\prime}=\frac{Q_{A^{z}}}{r}+O\left(\frac{1}{r}\right), \quad \bar{M}^{\prime}=\bar{M},
\end{gathered}
$$

where $\Delta^{\prime}$ is the square of the axial Killing vector and $J$ the total angular momentum.

The conditions at the horizon $\mathscr{H}$ are simply that $M^{\prime}$ has to attain a finite limit, specifically $\Delta_{\mathscr{H}}^{\prime}>0$ (distinguishing the horizon from the rotation axis). 
On the rotation axis $\Delta^{\prime}=\varrho^{2} O(1)$ and $\bar{M}^{\prime}$ tends to a finite limit quadratically with $\varrho$. The behaviour of $\tilde{\psi}^{\prime}$ and $A^{\prime}$ can be determined using Eqs. (3.5):

$$
\begin{gathered}
A^{\prime}= \pm Q_{A}+a(z) \varrho^{2}+O\left(\varrho^{4}\right), \\
\tilde{\psi}^{\prime}= \pm 4 J \mp Q_{A}^{T} \bar{Y}^{-1} a(z) \varrho^{2}+O\left(\varrho^{4}\right),
\end{gathered}
$$

with some function $a(z)$ and the signs referring to the upper and lower part of the axis.

\section{Static Single Black Holes}

Before we enter into the discussion of uniqueness theorems for black holes we want to prove another version of the "Generalized Lichnerowicz Theorem" (compare [13]). Although we have already given the more generally valid Theorem 2.8 in Sect. 2.2 we include it here, because it illustrates rather nicely the technique employed subsequently for black holes.

Theorem 6.1. (Generalized Lichnerowicz Theorem). Suppose $\Sigma_{3}$ with its metric $h_{a b}$ is topologically trivial and non-singular. If $M$ is a regular solution of $\nabla^{a}\left(M^{-1} \partial_{a} M\right)=0$ with the asymptotic behaviour $M=M_{0}+\frac{1}{r} M_{1}+O\left(\frac{1}{r^{2}}\right)$, then $M=M_{0}$ and $h_{a b}$ $=\delta_{a b}$, i.e. space-time is flat and empty.

Proof. Integrating $\nabla^{a}\left(M^{-1} \partial_{a} M\right)=0$ over $\Sigma_{3}$ we get

$$
\int_{r=\infty} M^{-1} \partial_{a} M d \Sigma^{a}=4 \pi M_{0}^{-1} M_{1}=0
$$

and hence $M_{1}=0$. As a consequence the total mass $m$ vanishes. From the "Positive Mass Theorem" [10] we conclude that the solution is the "vacuum" solution, i.e. Minkowski space with vanishing vector fields and constant scalars.

The static single black hole solutions can be completely determined in the present setting. It turns out that they are just the spherically symmetric ones already described in Sect. 4. For pure gravity (no vectors, no scalars) this is a classical result due to Israel [15]. Israel's argument was later improved and simplified by Robinson [43]. Israel was also able to generalize his theorem to the Einstein-Maxwell theory [44]. In this case his proof consists of two steps. First he shows that the electric potential $A$ is a function of the gravitational potential $\Delta$ and then he proceeds similarly to the case of pure gravity. Again there exists a simplified version of Israel's proof due to Bunting [11]. He makes use of the fact that the matrix of conserved gauge invariant currents $J_{a}=M^{-1} \partial_{a} M$ of the corresponding $S O(2,1) / S O(1,1) \sigma$-model has a component in the Lie algebra of $H=S O(1,1)$, namely $J_{a}^{H}=\Delta^{-1}\left[A \partial_{a} \Delta+\left(1-\Delta-A^{2}\right) \partial_{a} A\right]$, with vanishing "charge"

$\int_{r=\infty} J_{a}^{H} d \Sigma^{a}=0$. From that he is able to derive that the current $J_{a}^{H}$ has to vanish identically, which allows him to express $A$ as a function of $\Delta$. Then he uses an adaptation of the argument of Robinson to reach the desired conclusion. It is an interesting problem to generalize Bunting's method to the type of theories including scalars considered here. We shall, however, proceed somewhat differently using an argument put forward in [45] (compare also [46]). We use the fact 
that the vector (electrical) charges can be transformed to zero by a suitable Harrison transformation (compare Sect. 2). Then we show that the vector fields vanish identically if they carry no charges. In a second step we conclude that once the vector fields vanish, also the scalar fields have to be trivial, i.e. constant. This is expressed by the following theorems:

Theorem 6.2 (No Charges, no Vectors). For a static single black hole solution of Eqs. (2.16-18) with vanishing vector charges $Q_{A}=\frac{1}{4 \pi} \int_{r=\infty} \partial_{a} A d \Sigma^{a}=0$ the potentials $A$ are constant, and hence vanish identically being normalized to zero at infinity.

Proof. From Eq. (3.9b) we find

$$
\nabla^{a}\left(\Delta^{-1} A^{T} \hat{\bar{M}} \partial_{a} A\right)=\Delta^{-1} \partial^{a} A^{T} \hat{\bar{M}} \partial_{a} A
$$

and hence through integration

$$
\int_{\mathscr{H}} \Delta^{-1} A^{T} \hat{\bar{M}} \partial_{a} A d \Sigma^{a}=\int_{\Sigma_{3}} \Delta^{-1} \partial^{a} A^{T} \hat{\bar{M}} \partial_{a} A d \Sigma .
$$

(The surface term at infinity vanishes, since the integrand is $O\left(r^{-3}\right)$.) On the other hand

$$
\begin{aligned}
\int_{\mathscr{H}} \Delta^{-1} A^{T} \hat{\bar{M}} \partial_{a} A d \Sigma^{a} & =A_{\mathscr{H}}^{T} \int_{\mathscr{H}} \Delta^{-1} \hat{\bar{M}} \partial_{a} A d \Sigma^{a} \\
& =-A_{\mathscr{H}}^{T} \int_{r=\infty} \Delta^{-1} \hat{\bar{M}} \partial_{a} A d \Sigma^{a}=0,
\end{aligned}
$$

due to the boundary conditions on the horizon $\mathscr{H}$ and the vanishing of $Q_{A}$. Since $\hat{\bar{M}}$ is a positive matrix (compare Sect. 3 ) we get $\partial_{a} A=0$ as claimed.

Theorem 6.3 (No Vectors, no Scalars). If for a static single black hole solution of Eqs. (2.16-18) the vector potentials $A$ vanish, then the scalar fields are constant.

Proof. For vanishing vector potentials $A$ the scalar field equation Eq. (3.9d) reduces to

$$
\nabla^{a}\left(\bar{M}^{-1} \partial_{a} \bar{M}\right)=0
$$

Integrating we get

$$
\bar{Q}=\frac{1}{8 \pi} \int_{r=\infty} \bar{M}^{-1} \partial_{a} \bar{M} d \Sigma^{a}=0
$$

since the contribution at the horizon vanishes because $d \Sigma^{a}$ vanishes like $\Delta$ (compare Sect. 5).

On the other hand we can multiply Eq. (6.4) by $\bar{M}$ and obtain

$$
\nabla^{a} \partial_{a} \bar{M}=\partial^{a} \bar{M} \bar{M}^{-1} \partial_{a} \bar{M},
$$

and again by integration

$$
\int_{\Sigma_{3}} \partial^{a} \bar{M} \bar{M}^{-1} \partial_{a} \bar{M} d \Sigma=\int_{r=\infty} \partial_{a} \bar{M} d \Sigma^{a}=0 .
$$

From the positivity of $\bar{M}$ we deduce $\partial_{a} \bar{M}=0$ and therefore $\bar{M}=\bar{M}_{0}=\mathbf{1}$. 
Remark. As can be seen from the proof the assumption of staticity can be replaced by the weaker assumption of strict stationarity in this theorem.

Remark. For non-vanishing vector charges $Q_{A}$ we obtain from Eq. (3.9d),

$$
\nabla^{a}\left(\frac{1}{2} \bar{M}^{-1} \partial_{a} \bar{M}+\Delta^{-1} \frac{1}{\hat{\bar{c}}}\left(A \partial_{a} A^{T} \hat{\bar{M}}\right)_{\mathrm{pr}}\right)=0
$$

a relation between the $Q_{A}$ 's and the scalar charges $\bar{Q}$

$$
\bar{Q}=-\frac{1}{\hat{\bar{c}}}\left(A_{\mathscr{H}} Q_{A}^{T}\right)_{\mathrm{pr}} .
$$

Combining the two theorems with Israel's theorem for pure gravity we obtain the

Theorem 6.4. Any static single black hole solution of Eqs. (2.16-18) with a nondegenerate horizon is a member of the "Schwarzschild family", i.e. can be obtained from the Schwarzschild solution through a Harrison transformation.

This implies as a corollary the

Theorem 6.5. (Generalized Israel Theorem). All static single black hole solutions of Eqs. (2.16-18) are spherically symmetric.

Proof. Starting from an arbitrary single black hole solution of Eqs.(2.16-18) we can transform it via a Harrison transformation (compare Sect. 3) into one with vanishing $A$ and constant $\bar{M}$. It follows from Eqs. (3.10-15) that this transformation does not change the boundary conditions at the horizon. Since Eqs. (3.8-9) reduce to those for pure gravity for vanishing $A$ and constant $\bar{M}$, we conclude that the transformed function $\Delta$ has to be the Schwarzschild solution. Reversing the transformation, we obtain the solution we started with as the Harrisontransformed Schwarzschild solution, exhibiting at the same time its spherical symmetry.

\section{Stationary, Axisymmetric, Single Black Holes}

The prototype of a stationary, axisymmetric black hole is the Kerr solution, depending on two parameters, mass and angular momentum. This is, in vacuum, the only such single black hole solution [47]. Also for the Einstein-Maxwell theory the questions of existence and uniqueness have a simple, positive answer in the form of the Kerr-Newman solution, depending on four parameters - mass, angular momentum, electric and magnetic charge. The uniqueness of the Kerr-Newman solution has only rather recently been proven by Mazur and Bunting $[12,11]$. From our experience with the static solutions we expect the existence problem to be more delicate when we turn to the theories considered here. In fact, in the static case the charges $\bar{Q}$ for the scalars cannot be given freely, but are determined by the vector charges $Q_{A}$. On the other hand we were able to show that all the static single black holes are members of the "Schwarzschild family", i.e. could be generated from the Schwarzschild solution via a suitable Harrison transformation acting on 
$M$. It is tempting to speculate that this situation prevails in the case of rotating black holes, if we replace Schwarzschild by Kerr. That this speculation can be materialized will be shown below.

In order to prove the uniqueness of the solutions with given $v$ ("irreducible mass" or size of the horizon), angular momentum $J$ and charges $Q_{A}$ we shall generalize the uniqueness theorem of Mazur [12]. As already observed by Mazur himself [48] his theorem can in fact be rather easily extended to theories of the type considered here, the essential ingredient being the 3-dimensional $\sigma$-model structure for axisymmetric solutions. In contrast to the uniqueness proofs for static black holes using the $G / H \sigma$-model, the Mazur theorem uses the $G / H^{\prime} \sigma$-model based on the matrix $M^{\prime}$ instead of $M$. This is necessary because only $G / H^{\prime}$ has positive definite metric as required in the proof of the theorem. Moreover this formulation avoids the singularities of the matrix $M$ at the ergosurface.

As discussed in Sect. 5 we can choose canonical coordinates $\varrho$ and $z$ such that the horizon meets the rotation axis at the two points $z= \pm v$. The horizon itself is then given by $\mathscr{H}=\{\varrho=0,|z| \leqq v\}$. The exterior region of the black hole is the union of the two pieces of the rotation axis $\{\varrho=0,|z|>v\}$ with $\Sigma_{2}=\{(z, \varrho): \varrho>0\}$.

Theorem 7.1 (Mazur). Two solutions $M_{i}^{\prime}=\left(P_{i}^{\prime}\right)^{+} P_{i}^{\prime}(i=1,2)$ of the equation $\nabla\left(\varrho\left(M_{i}^{\prime}\right)^{-1} \partial M_{i}^{\prime}\right)=0$ taking their values in the Riemannian symmetric space $G / H^{\prime}$ and obeying the same boundary conditions for single black holes (i.e. equal values for $v, J$ and $Q_{A}$ ) are identical.

Proof. Putting $J_{i}=\frac{1}{2}\left(M_{i}^{\prime}\right)^{-1} \partial M_{i}^{\prime}$ and using $\nabla\left(\varrho J_{i}\right)=0$ and $J_{i}^{+}=M_{i}^{\prime} J_{i}\left(M_{i}^{\prime}\right)^{-1}$, we find

$$
\begin{aligned}
\frac{1}{4} \nabla\left(\varrho \operatorname{Tr} \partial\left(\left(M_{1}^{\prime}\right)^{-1} M_{2}^{\prime}\right)\right) & =\frac{1}{2} \nabla\left(\varrho \operatorname{Tr}\left(\left(M_{1}^{\prime}\right)^{-1} M_{2}^{\prime}\left(J_{2}-J_{1}\right)\right)\right) \\
& =\varrho \operatorname{Tr}\left(\left(M_{1}^{\prime}\right)^{-1}\left(J_{2}-J_{1}\right)^{+} M_{2}^{\prime}\left(J_{2}-J_{1}\right)\right) \\
& =\varrho \operatorname{Tr}\left(\mathscr{I}_{12}^{+} \mathscr{F}_{12}\right)
\end{aligned}
$$

with $\mathscr{J}_{12} \equiv P_{2}^{\prime}\left(J_{2}-J_{1}\right)\left(P_{1}^{\prime}\right)^{-1}$. Integrating this identity over $\Sigma_{2}$ and using $\operatorname{Tr} J_{i}=0$, we obtain

$$
\left.\begin{array}{rl}
\int_{\Sigma_{2}} \operatorname{Tr}\left(\mathscr{I}_{12}^{+} \mathscr{I}_{12}\right) \varrho d \varrho d z & =\int_{\partial \Sigma_{2}} \frac{1}{2} \operatorname{Tr}\left(\left(M_{1}^{\prime}\right)^{-1} M_{2}^{\prime}\left(J_{2}-J_{1}\right)\right) \varrho d n \\
& \equiv \int_{\partial \Sigma_{2}} \frac{1}{2} \operatorname{Tr}\left(\left(\left(P_{2}^{\prime}\left(P_{1}^{\prime}\right)^{-1}\right)^{+}-P_{1}^{\prime}\left(P_{2}^{\prime}\right)^{-1}\right) \mathscr{I}_{12}\right) \varrho d n .
\end{array}\right\}
$$

In order to evaluate the boundary term we use the boundary conditions discussed in Sect. 5. There is no contribution from the horizon due to the explicit factor $\varrho$. At infinity one finds $P_{2}^{\prime}\left(P_{1}^{\prime}\right)^{-1}=\mathbf{1}+O\left(\frac{1}{r}\right)$ and the normal component of $\mathscr{I}_{12}$ is $O\left(\frac{1}{r^{2}}\right)$; hence the boundary term at infinity vanishes for any two solutions. In order to analyze the contribution from the axis we need the detailed form of the boundary conditions [compare Eq. (5.11)]. For two solutions with the same angular momentum $J$ and vector charges $Q_{A}$ it follows that both $P_{2}^{\prime}\left(P_{1}^{\prime}\right)^{-1}$ and $\mathscr{I}_{12}$ stay finite at the axis. Hence the boundary term at the axis vanishes as well. This implies $\mathscr{I}_{12}=0$, i.e., $\partial\left(\left(M_{1}^{\prime}\right)^{-1} M_{2}^{\prime}\right)=0$ leading to $M_{1}^{\prime}=M_{2}^{\prime}$ since $\left(M_{1}^{\prime}\right)^{-1} M_{2}^{\prime} \rightarrow \mathbf{1}$ for $r \rightarrow \infty$. 
As an immediate consequence of this theorem a stationary, axisymmetric black hole with vanishing vector charges $Q_{A}$ is necessarily a Kerr solution. This is the analogue of the "No charges, no vectors" and "No vectors, no scalars" theorems proven for static black holes in Sect. 6 .

Remark. It is also possible to generalize Bunting's method [11] to prove uniqueness exploiting the harmonicity of the map $M^{\prime}: \mathbf{R}^{2} \rightarrow G / H^{\prime}$.

The action (A.10) of the group $G$ transforms solutions of Eqs. (3.8-9) into solutions. The requirement to preserve the asymptotic behaviour (5.1), is, however, only satisfied by elements of the subgroup $H \subset G$ which is the isotropy group of $M=1$ in $G / H$. Applying this "Ehlers-Harrison" group $H$ to the Kerr solutions (embedded into the $G / H \sigma$-model) produces the "Kerr-family". What remains to be shown is that the "Kerr-family" contains black holes with arbitrary values of $v, J$ and $Q_{A}$. This is expressed by the following proposition proved in [49]:

\section{Proposition 7.2.}

1. Acting on a Kerr solution with an element of $H$ which does not produce a NUT-charge yields a black hole solution satisfying the boundary conditions (5.10-11).

2. Applying these transformations to all Kerr holes one obtains black holes with arbitrary values of $v, J$ and $Q_{A}$.

Remark. The proof of this proposition is non-trivial because the group $G$ acts in a simple way only on $M$ whereas the black hole boundary conditions involve $M^{\prime}$.

Combining the Mazur Theorem with Proposition 7.2 yields immediately the announced result concerning the "Kerr-family".

Theorem 7.3. Any stationary, axisymmetric single black hole solution of Eqs. (2.16-18) is a member of the "Kerr-family", i.e. can be obtained through a Harrison transformation from the Kerr solution.

\section{Stationary Multi-Black Holes}

Single black holes have many analogies with solitons known from certain 2-dimensional non-linear field theories. Therefore one might expect a particle-like behaviour for configurations containing several black holes - at least if they are far apart and slowly moving. Among other things that would mean that such configurations could be well described by a finite number of parameters, such as masses, charges and positions. Clearly this would require that emitted or absorbed radiation could be neglected to lowest order. Some preliminary steps in this direction are described in [50].

In view of the highly non-linear character of the field equations and their relativistic covariance the effective dynamics of such "particles" is expected to be rather involved. Therefore it is highly remarkable that there even exist static multiblack hole configurations. The best known example is a superposition of several extremal Reissner-Nordstrøm holes, contained in the Majumdar-Papapetrou (M-P) class of solutions [9]. The existence of such a configuration of several black 
holes in indifferent equilibrium may be attributed to a complete balance between their gravitational attraction and electric repulsion. The explicit formula for the solution with $N$ holes is

$$
\begin{gathered}
\Delta=(1-m \tau)^{-2}, \quad \Phi=m \tau(1-m \tau)^{-1}, \\
\tau=-\frac{1}{m} \sum_{i=1}^{N} \frac{m_{i}}{r_{i}}, \quad r_{i}=\left|x-x_{i}\right|, \quad m=\sum m_{i} .
\end{gathered}
$$

Just as for the single hole, the corresponding harmonic map from $\Sigma_{3}$ to the symmetric space $\Phi=S O(2,1) / S O(1,1)$ can be factorized via some harmonic function $\tau$ on $\Sigma_{3}$ and a light-like geodesic in $\Phi$. The metric $h_{a b}$ on $\Sigma_{3}$ is again flat. The question arises if the M-P type solutions are the only stationary multi-black hole solutions. There is a rotating version of the extremal Reissner-Nordstrøm solution, the extremally charged (i.e. $q^{2}=m^{2}$ ) Kerr-Newman solution [13], which does, however, not describe a black hole but a naked singularity. Similarly there are rotating versions of the M-P solutions, the Perjes-Israel-Wilson (P-I-W) solutions [51], but as to be expected they have naked singularities too [52]. The P-I-W class exhausts the completely light-like solutions, but it is not known, if equilibrium configurations of black holes can only exist for extremally charged ones. For the static case, in vacuum, it has recently been proven that there are no multi-black holes [14], which is intuitively obvious (but nevertheless hard to prove) as there is no force to balance gravitational attraction. For rotating black holes it could in principle be possible to balance the mass attraction by the spin repulsion. In fact, it is possible to construct explicitly so-called multi-Kerr solutions using Bäcklund transformations [53], but none of them seems to be free of naked singularities.

In the following we shall discuss generalizations of the extremally charged solutions for the Einstein-Maxwell theory to the type of theories considered here.

Definition 8.1. A solution of Eqs. (3.8-9) is called extremally charged, if the image in $\Phi$ is completely light-like, i.e. if $\left\langle M^{-1} \partial_{a} M, M^{-1} \partial_{b} M\right\rangle=0$.

The term "extremally charged" for this type of solutions is justified by the relation (generalizing the relation $q^{2}=m^{2}$ for the extremal Reissner-Nordstrøm solution)

$$
m^{2}+\frac{1}{4} n^{2}+\frac{1}{2}\langle\bar{Q}, \bar{Q}\rangle-\frac{1}{2} Q_{A}^{T} Q_{A}=0
$$

obtained from the defining relation by picking its leading term for $r \rightarrow \infty$. For the spherically symmetric case this relation implies that the surface gravity vanishes. In the thermodynamic interpretation of black holes [54] vanishing surface gravity means zero temperature. In fact, spherical symmetry is not essential as shown by the

Theorem 8.2. For extremally charged, strictly stationary solutions the surface gravity $\kappa^{2}=\frac{1}{4} h^{a b} \partial_{a} \Delta \partial_{b} \Delta$ vanishes.

Proof. For strictly stationary solutions the boundary condition $T_{\alpha \beta} \alpha^{\alpha} l^{\beta}=0$ on the horizon turns into

$$
T_{\alpha \beta} K^{\alpha} K^{\beta}=\frac{1}{2} \Delta h^{a b}\left(\partial_{a} A^{T} \hat{\bar{M}} \partial_{b} A+\Delta\left\langle\bar{J}_{a}, \bar{J}_{b}\right\rangle\right)=0 .
$$


However from $R_{a b}=0$ we find

$$
h^{a b} \partial_{a} \Delta \partial_{b} \Delta=h^{a b}\left(2 \Delta \partial_{a} A^{T} \hat{\bar{M}} \partial_{b} A-\omega_{a} \omega_{b}-2 \Delta^{2}\left\langle\bar{J}_{a}, \bar{J}_{b}\right\rangle\right) .
$$

In view of Eq. (8.3) and the boundary condition for $\omega_{a}$, the right-hand side of Eq. (8.4) vanishes on the horizon.

For such extremally charged solutions $h_{a b}=\delta_{a b}$ (in suitable coordinates), since due to Eq. (3.8) the Ricci tensor and hence also the Riemann tensor of $h_{a b}$ vanishes. The simplest case is again given in terms of a light-like geodesic and a single harmonic function as for the M-P solutions. These are determined by some lightlike tangent vector to $\Phi$ at $M_{0}$ - the asymptotic value of $M$ at $r=\infty$ - and a single harmonic function $\tau(x)$ on $\Sigma_{3}$. What are the requirements on that tangent vector and the harmonic function $\tau(x)$ in order to obtain a superposition of $N$ black holes? In order to avoid a NUT charge, i.e. a $\frac{1}{r^{2}}$ contribution to the current $\Delta^{-2} \omega_{a}$, this current has to vanish identically; hence the solution has to be static. The additional requirements concerning the behaviour of $\bar{M}$ at $\tau=-\infty$ have already been discussed in Sect. 4.

Next we have to study the conditions on the harmonic function $\tau(x)$ on $\mathbf{R}^{3}$. As discussed by Hartle and Hawking [52] the only acceptable solution for a static superposition of $N$ black holes is $\tau(x)=-\frac{1}{m} \sum_{i=1}^{N} \frac{m_{i}}{r_{i}}$ as for the multi-ReissnerNordstrøm solution. It is easy to see that the $m_{i}$ have to be non-negative in order to avoid naked singularities.

There is an obvious generalization of the solutions discussed above, involving several harmonic functions, based on the composition theorem on harmonic and totally geodesic maps mentioned earlier. Let $\tau=\left(\tau_{1}, \ldots, \tau_{n}\right)$ be a harmonic map from $\Sigma_{3}=\mathbf{R}^{3}$ to $\mathbf{R}^{n}$, given in terms of the $n$ harmonic functions $\tau_{i}(x)$ and $\hat{\phi}$ a totally geodesic map from $\mathbf{R}^{n}$ into $G / H$. Then the composition $\hat{\phi} \circ \tau$ defines again a harmonic map from $\Sigma_{3}$ into $G / H$. The tangent vectors to the image of $\hat{\phi}$ in $G / H$ at some point constitute a subset of the part of the Lie algebra of $G$ corresponding to $G / H$. Since $\hat{\phi}$ is totally geodesic, it maps straight lines in $\mathbf{R}^{n}$ into geodesics of $G / H$. It is easy to see that this implies that the image of $\hat{\phi}$ is generated by an abelian subalgebra, hence a flat submanifold of $G / H$.

In order to obtain extremally charged solutions we have to require in addition that this abelian subalgebra is completely light-like, i.e. all its elements are lightlike. For a solution describing a superposition of black holes there are in general further constraints arising from the finiteness of the scalar fields at the horizon. It seems that this requires that the various harmonic functions $\tau_{i}(x)$ have common singularities, but no proof of that is known to us. An example demonstrating this behaviour is given in [41].

\section{Open Problems}

In this chapter we wish to conclude with a list of open problems. We begin with the vacuum case. 
Vacuum Case. For static solutions the situation is rather satisfactory. In the stationary case the most important unresolved question is whether a non-rotating hole need be axisymmetric. If not there are new single black hole solutions to be found. The other outstanding problem is whether there are stationary solutions with more than one black hole. Experience with the "multiple Kerr" solutions obtained via the Inverse Scattering Method [53] suggests a negative answer.

Einstein-Maxwell. In the electromagnetic case there remains the problem whether there exist topologically non-trivially configurations without horizons. In the case of static holes one would like to know whether the only multi-black hole solutions are the Papapetrou-Majumdar metrics. In the stationary case one inherits the open problems in the vacuum case together with the additional one of whether a rotating black hole need be axisymmetric.

General Case. In the case of the more general theories, in addition to the problems inherited from the two previous cases the classification of the solutions based on totally light-like totally geodesic submanifolds of $G / H$ is not yet clear.

Three additional types of problems also suggest themselves:

1) Whether stationarity or axisymmetry of the metric implies stationarity or axisymmetry of the matter fields under the global conditions.

2) The extension to different boundary conditions - for example those arising from a cosmological term [16] or asymptotically Melvin type boundary conditions [55].

3) The extension to black holes in arbitrary dimensions.

\section{Appendix A. Non-Linear $\sigma$-Models and Symmetric Spaces}

In this appendix we shall study group theoretical aspects of non-linear $\sigma$-models with a target space $\Phi$ which is a non-compact Riemannian or pseudo-Riemannian symmetric space $G / H$.

\section{A.1. Non-Compact Riemannian Symmetric Spaces}

Let $G$ be a non-compact real form of some-compact Lie group. There is an involutive automorphism $\tau: G \rightarrow G, \tau^{2}=1$ such that

$$
H=\{h \in G: \tau(h)=h\}
$$

is the maximal compact subgroup of $G$ and the coset space $G / H$ is a non-compact Riemannian symmetric space $[30,31,56]$.

In order to parametrize the coset space $G / H$ we can choose a group element $P$ as representative for each coset, i.e. introduce fields $P(x)$,

$$
P: \Sigma \ni x \rightarrow P(x) \in G .
$$

Customarily, $G / H$ denotes the space of left cosets $g H$. For purely historical reasons we use the space of right cosets which is sometimes denoted by $H \backslash G$. As these two spaces are isomorphic we prefer the notation $G / H$. The choice (A.2) of representatives is obviously not unique and the freedom to choose representatives leads to a gauge invariance with gauge group $H$, in addition to the group action of $G$ on $G / H$,

$$
P(x) \rightarrow h(x) P(x) g^{-1}, \quad h(x) \in H, \quad g \in G .
$$


The field equations for $P$ (and the action) are invariant under the action of both $G$ and $H$. Because of this gauge invariance the true dynamical variables have their values in the coset space $G / H$.

We can eliminate the gauge group $H$ by choosing one standard representative for each coset. We will obtain a particularly simple parametrization of $G / H$ [57] if we choose a solvable subgroup (represented by matrices "triangular" in the sense of the Iwasawa decomposition [30]) which intersects each coset once. Given such a gauge choice (or any other one) the action (A.3) of a $g \in G$ will lead to non-standard representatives and must therefore be accompanied by an induced gauge transformation $h(P(x), g) \in H$ (depending in general non-linearly on $P$ and $g$ ) in order to maintain the gauge choice

$$
P(x) \rightarrow h(P(x), g) P(x) g^{-1}, \quad g \in G .
$$

In order to construct the metric $\gamma_{i j}$ we consider the 1 -form $d P(x) P(x)^{-1}$ with value in the Lie algebra $\mathscr{G}$ of $G$ and its decomposition

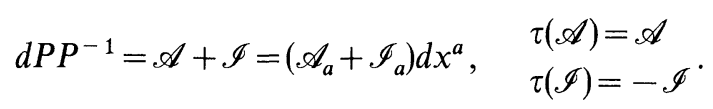

The transformation laws [induced by (A.3)] for $\mathscr{A}$ and $\mathscr{I}$ are

$$
\begin{gathered}
\mathscr{A}=\frac{1}{2}\left(d P P^{-1}+\tau\left(d P P^{-1}\right)\right) \rightarrow h \mathscr{A} h^{-1}+d h h^{-1}, \\
\mathscr{I}=\frac{1}{2}\left(d P P^{-1}-\tau\left(d P P^{-1}\right)\right) \rightarrow h \mathscr{I} h^{-1},
\end{gathered}
$$

i.e. $\mathscr{A}$ can be interpreted as connection for $H$ whereas $\mathscr{I}$ transforms $H$-covariantly and both are $G$-invariant. Given any invariant scalar product $\langle\cdot, \cdot\rangle$ on $\mathscr{G}$ we can define an invariant metric $\gamma$ on $G / H$ by

$$
d \phi^{i} d \phi^{j} \gamma_{i j}(\phi) \equiv\langle\mathscr{I}, \mathscr{I}\rangle .
$$

If $G$ is simple any such $\langle\cdot, \cdot\rangle$ is a (positive) multiple of the Killing metric and for each faithful representation $\varrho: \mathscr{G} \ni \mathscr{I} \rightarrow \hat{I} \equiv \varrho(\mathscr{I})$ there is a (positive) constant $\hat{c}$ such that

$$
\langle\mathscr{I}, \mathscr{I}\rangle=\hat{c} \operatorname{Tr}(\hat{\mathscr{I}} \hat{\mathscr{I}}) .
$$

Note that the scalar product $\langle\cdot, \cdot\rangle$ on $\mathscr{G}$ is indefinite but the metric $\gamma$ on $G / H$ is (positive) definite.

If $G=\prod_{l} G_{l}$ is semi-simple there are for each faithful representation $\varrho$ constants $\hat{c}_{l}$ such that

$$
\langle\mathscr{I}, \mathscr{I}\rangle=\sum_{l} \hat{c}_{l} \operatorname{Tr}\left(\hat{\mathscr{I}}_{l} \hat{\mathscr{I}}_{l}\right),
$$

where $\mathscr{I}_{l}$ is the component of $\mathscr{I}$ in $\mathscr{G}_{l}$ and $\widehat{\mathscr{I}}_{l} \equiv \varrho\left(\mathscr{I}_{l}\right)$. We will, however, use the simpler notation of Eq. (A.7) for this case as well. The situation remains essentially unchanged if $G$ contains one abelian factor, but becomes more complicated in the presence of several such factors $(U(1)$ or $S O(1,1) \sim \mathbf{R})$.

Given an $r$-dimensional faithful representation $\varrho$ of $\mathscr{G}$ we can project any $r \times r$ matrix $\hat{T}$ onto $\mathscr{G}, \hat{T} \rightarrow(\hat{T})_{\text {pr }}$, such that for every $\mathscr{I} \in \mathscr{G}$,

$$
\left\langle(\hat{T})_{\mathrm{pr}}, \mathscr{I}\right\rangle=\hat{c} \operatorname{Tr}(\hat{T} \hat{\mathscr{I}})
$$

and thus $(\widehat{\mathscr{I}})_{\mathrm{pr}}=\mathscr{I}$. This projection satisfies $\left(\hat{\mathrm{g}} \widehat{T} \hat{\mathrm{g}}^{-1}\right)_{\mathrm{pr}}=g(\widehat{T})_{\mathrm{pr}} g^{-1}$ for all $g \in G$. 
The automorphism $\tau$ provides us with a canonical embedding of $G / H$ in $G$ [59],

$$
P \rightarrow M=\tau\left(P^{-1}\right) P, \quad \tau(M)=M^{-1},
$$

where $M$ is $H$-invariant and transforms covariantly under $G$

$$
M \rightarrow \tau(g) M g^{-1}, \quad g \in G .
$$

For the corresponding current $J=\frac{1}{2} M^{-1} d M$ we find

$$
\mathscr{I}=D P P^{-1}=\frac{1}{2} P M^{-1} d M P^{-1} \equiv P J P^{-1},
$$

where $D P=d P-\mathscr{A} P$ is the $H$-covariant derivative of $P$.

The line element (A.6) on $G / H$ can be reexpressed in terms of $M$,

$$
d \phi^{i} d \phi^{j} \gamma_{i j}(\phi)=\frac{1}{4}\left\langle M^{-1} d M, M^{-1} d M\right\rangle,
$$

and the $\sigma$-model field equations can be rewritten in the equivalent forms

$$
D^{\alpha} \mathscr{I}_{\alpha}=0 \quad \text { or } \quad \nabla^{\alpha} J_{\alpha}=0 \quad \text { or } \quad \nabla^{\alpha}\left(M^{-1} \partial_{\alpha} M\right)=0,
$$

where $D_{\alpha} \mathscr{I}_{\beta}=\nabla_{\alpha} \mathscr{I}_{\beta}-\left[\mathscr{A}_{\alpha}, \mathscr{I}_{\beta}\right]$. There are $\operatorname{dim} G$ currents $J$ and field equations $\nabla J=0$, but clearly only $\operatorname{dim} G / H$ of them are independent. In fact, the currents $J$ obey the identiy $\tau(J)=-M J M^{-1}$ due to $\tau(M)=M^{-1}$.

We will choose the basis for a representation $\varrho$ such that $\tau(\hat{g})=\left(\hat{g}^{+}\right)^{-1}$ for all $g \in G$, and thus $\tau(\hat{\mathscr{I}})=-\hat{\mathscr{I}}^{+}$for all $\mathscr{I} \in \mathscr{G}$. In such a basis the matrix $\hat{M} \equiv \varrho(M)$ $=\hat{P}^{+} \hat{P}$ is hermitian and positive definite.

Note that $M$ and $P$ are very close analogues of the metric and the moving frame (tetrad) in general relativity. We see that the "metric" $M$ is sufficient to formulate the $\sigma$-model and this remains true if we include vector fields. Nevertheless the "moving frame" $P$ with a "triangular" gauge choice yields a very convenient and simple parametrization of $G / H$. The situation changes if we add fermion fields to some of our models (e.g. $N=1$ supergravity) because these fermion fields transform with some representation of $H$ and not of $G$. The "moving frame" $P$ is, therefore, necessary in order to describe these fermions.

\section{A.2. Pseudo-Riemannian Symmetric Spaces}

The situation becomes slightly more complicated if we consider a pseudoRiemannian symmetric space $G / H$ [56]. In this case we have a non-compact Lie group $G$ and an involutive automorphism $\tau$ which defines the subgroup $H$ of $G$ as before. In addition there is a different involutive automorphism $\tau^{\prime}$ commuting with $\tau\left(\tau \tau^{\prime}=\tau^{\prime} \tau=\tau^{\prime \prime}\right)$ which determines the maximal compact subgroup $H^{\prime}$ of $G$

$$
H^{\prime}=\left\{h \in G: \tau^{\prime}(h)=h\right\} .
$$

Again we can choose $P$, decompose $d P P^{-1}$, and construct $M$ and $\gamma$ as before. The basis for a representation $\varrho$ will be chosen such that $\tau(\hat{g})=\hat{X}^{-1}\left(\hat{g}^{+}\right)^{-1} \hat{X}$ with a hermitian matrix $\hat{X}$. If we define $\hat{M} \equiv \hat{X} \varrho(M)=\hat{P}^{+} \hat{X} \hat{P}$, the matrix $\hat{M}$ will again be hermitian. Due to the non-compactness of $H$ the matrix $\hat{X}$ and thus $\hat{M}$ and the metric $\gamma$ will, however, not be positive definite. 
Finally there are subgroups

$$
\begin{gathered}
H^{\prime \prime}=\left\{h \in G: \tau^{\prime \prime}(h)=h\right\}, \\
K=\left\{k \in H^{\prime}: \tau(k)=k\right\} \equiv H \cap H^{\prime},
\end{gathered}
$$

where $K$ is the maximal compact subgroup of $H$ and $H^{\prime \prime} / K$ is a Riemannian symmetric space which is a totally geodesic subspace [30] of both $G / H$ and $G / H^{\prime}$ (in fact the maximal such space because $H^{\prime \prime} / K=G / H \cap G / H^{\prime}$ ).

\section{A.3. Consistent Truncations}

Given a non-linear $\sigma$-model with target space $\Phi$ and metric $\gamma$ we may be interested to study a truncated $\sigma$-model with target space $\tilde{\Phi} \subset \Phi$ and the induced metric $\tilde{\gamma}=\left.\gamma\right|_{\tilde{\Phi}}$

Definition A.1 (Consistent Truncation). A non-linear $\sigma$-model with target space $\widetilde{\Phi}$ is a consistent truncation of another $\sigma$-model with target space $\Phi$ if $\widetilde{\Phi} \subset \Phi$ and if every solution of the field equations $(2.2-3)$ for the $\widetilde{\Phi} \sigma$-model is a solution of the field equations for the $\Phi \sigma$-model as well.

This is the case iff $\widetilde{\Phi}$ is a totally geodesic subspace of $\Phi$.

If $\Phi$ is a Riemannian symmetric space $G / H$, then every totally geodesic subspace of $\Phi$ is again a Riemannian symmetric space $\widetilde{G} / \tilde{H}$. The automorphism $\tau$ maps the subgroup $\widetilde{G} \subset G$ onto itself, $\tilde{\tau}=\left.\tau\right|_{\tilde{G}}$, and therefore $H \cap \widetilde{G}=\widetilde{H} \subset H$. Similar statements hold true if $\Phi$ is a pseudo-Riemannian symmetric space.

\section{Appendix B. A List of $\sigma$-Models}

In Sect. 2.1 we have studied a rather general class of non-linear $\bar{G} / \bar{H} \sigma$-models coupled to $k$ vectors and gravity. In Sect. 3.1 we have shown how solutions of such theories with a Killing vector field give rise to dimensionally reduced, 3-dimensional theories consisting of a non-linear $\sigma$-model with target space $\Phi$ coupled to (3-dimensional) gravity. The metric on the $(\operatorname{dim} \bar{G} / \bar{H}+2 k+2)$-dimensional space $\Phi$ is positive definite or indefinite with $2 k$ negative contributions if the Killing vector is space-like $(\Delta<0)$ or time-like $(\Delta>0)$ respectively. Finally we have observed that, depending on the details of the 4-dimensional theory, it may or may not be true that $\Phi$ is a symmetric space $G / H^{\prime}$ respectively $G / H$. If, however, $\Phi$ is a symmetric space then $G / H^{\prime}$ is a non-compact Riemannian symmetric space and $G / H$ is a Pseudo-Riemannian form of $G / H^{\prime}$ [compare Eqs. (3.6-9)]. In this appendix we shall give a complete list of all the cases where $\Phi$ is indeed a symmetric space. We shall also give a list of some possible static truncations (compare Sect. 2.2).

In order to reduce the list of all possible symmetric spaces $G / H$ we can first exclude as uninteresting all the cases where $G / H$ is a direct product $\left(G_{1} / H_{1}\right)$ $\times\left(G_{2} / H_{2}\right)$. This can happen only if $\bar{G} / \bar{H}$ is a direct product of the form $\left(\bar{G}_{1} / \bar{H}_{1}\right)$ $\times\left(G_{2} / H_{2}\right)$ and the action of $G_{2}$ on the vectors is trivial. In this situation either the scalars parametrizing $G_{2} / H_{2}$ are constant or there are naked singularities (Theorem 6.1 applied to $G_{2} / H_{2}$ ). Moreover $G$ must contain the subgroup $S L(2)$ and can therefore not be abelian. Thus $G$ must be a non-compact real form of a semi-simple Lie group $G_{\text {co }}$ [30]. From the list of all simple pseudo-Riemannian symmetric spaces given in [56] we find the following possibilities: 
i) $G=Q^{c} \times Q^{c}, H=Q^{c}, H^{\prime}=Q \times Q$, where $Q$ is a compact simple Lie group with complexification $Q^{c}$;

ii) $G=Q^{c}, H=Q_{\mathrm{nc}}, H^{\prime}=Q$, where $Q_{\mathrm{nc}}$ is a non-compact real form of $Q$;

iii) $G=Q^{c}, H=R^{c}, H^{\prime}=Q$, where $R$ is the maximal compact subgroup of $Q_{\mathrm{nc}}$;

iv) $G=Q_{\mathrm{nc}} \times Q_{\mathrm{nc}}, H=Q_{\mathrm{nc}}, H^{\prime}=R \times R$;

v) $G_{\mathrm{co}}$ is simple. There are 56 pseudo-Riemannian symmetric spaces $G / H$ for the exceptional groups. For the classical groups there are 21 series of such spaces depending on one parameter, 12 series depending on two parameters and 3 series depending on four parameters.

We shall now require the additional properties found in Sect. 3.1 in order to reduce this list in three steps.

Step 1. $H$ must be a non-compact real form of the maximal compact subgroup $H^{\prime}$ of $G$. This excludes the cases iii), iv) and reduces case v) to 22 exceptional coset spaces, 5 series with one parameter, 4 series with two parameters and 3 series with three parameters, whereas, e.g., all the following examples are excluded:

\begin{tabular}{lll}
\hline$G$ & $H$ & $H^{\prime}$ \\
\hline$F_{4(+4)}$ & $S O(5,4)$ & $U S p(6) \times S U(2)$ \\
$E_{7(-5)}$ & $S U(6,2)$ & $S O(12) \times S O(3)$ \\
$S U^{*}(2 n)$ & $S O^{*}(2 n)$ & $U S p(2 n)$ \\
$S O(n, n)$ & $G L(n)$ & $S O(n) \times S O(n)$ \\
$S U(p, q)$ & $S O(p, q)$ & $S(U(p) \times U(q))$ \\
\hline
\end{tabular}

Moreover the four parameter family $G=S O(p+q, r+s), H=S O(p, r) \times S O(q, s)$, $H^{\prime}=S O(p+q) \times S O(r+s)$ is reduced to a three parameter family by the restriction $r=s$.

Step 2. The intersection $G / H \cap G / H^{\prime} \equiv H^{\prime \prime} / K$ must be the direct product of the two spaces $S L(2) / S O(2)$ and $\bar{G} / \bar{H}$. This excludes cases i), ii). The list for case v) is restricted further and again all the following examples are excluded (at least for general values of the parameters).

\begin{tabular}{lll}
\hline$G$ & $H$ & $H^{\prime \prime}$ \\
\hline$F_{4(+4)}$ & $U S p(4,2) \times S U(2)$ & $S O(5,4)$ \\
$E_{7(-5)}$ & $S O(8,4) \times S O(3)$ & $S O(8,4) \times S O(3)$ \\
$S O^{*}(4 n)$ & $S U^{*}(2 n) \times S O(1,1)$ & $S U^{*}(2 n) \times S O(1,1)$ \\
$S L(p+q)$ & $S O(p, q)$ & $S(G L(p) \times G L(q))$ \\
$S U(p+q, p+r)$ & $S(U(p, r) \times U(q, p))$ & $S(U(p, p) \times U(q, r))$ \\
\hline
\end{tabular}

Step 3. The generators of the coset space $G / H^{\prime \prime}$ have to transform under the 2-dimensional representation of $S L(2) \subset H^{\prime \prime}$ in order to reproduce the scale transformation (3.12). The possibility

$$
\frac{G}{H}=\frac{S O(n+2, n+1)}{S O(n, 1) \times S O(2, n)}, \quad H^{\prime \prime}=S O(n, n) \times S O(2,1)
$$

is ruled out by this last condition. 
Table 2. List of all symmetric spaces obtained by dimensional reduction from four to three dimensions of theories with scalars and vectors

\begin{tabular}{|c|c|c|c|c|}
\hline$\#$ & $G / H$ & $\bar{G} / \bar{H}$ & $\operatorname{dim} \bar{G} / \bar{H}$ & $k$ \\
\hline 1 & $S L(n+2) / S O(n, 2)$ & $G L(n) / S O(n)$ & $\frac{1}{2} n(n+1)$ & $n$ \\
\hline 2 & $\frac{S U(p+1, q+1)}{S(U(p, 1) \times U(1, q))}$ & $U(p, q) / U(p) \times U(q)$ & $2 p q$ & $p+q$ \\
\hline 3 & $\frac{S O(p+2, q+2)}{S O(p, 2) \times S O(2, q)}$ & $\frac{S O(p, q)}{S O(p) \times S O(q)} \times \frac{S O(2,1)}{S O(2)}$ & $p q+2$ & $p+q$ \\
\hline 4 & $S O^{*}(2 n+4) / U(n, 2)$ & $\frac{S O^{*}(2 n) \times S U(2)}{U(n) \times S U(2)}$ & $\frac{1}{2} n(n-1)$ & $2 n$ \\
\hline 5 & $S p(2 n+2 ; \mathbf{R}) / U(n, 1)$ & $S p(2 n ; \mathbf{R}) / U(n)$ & $\frac{1}{2} n(n+1)$ & $n$ \\
\hline 6 & $\frac{G_{2(+2)}}{S U(1,1) \times S U(1,1)}$ & $S U(1,1) / U(1)$ & 2 & 2 \\
\hline 7 & $\frac{F_{4(+4)}}{S p(6 ; \mathbf{R}) \times S U(1,1)}$ & $S p(6 ; \mathbf{R}) / U(3)$ & 12 & 7 \\
\hline 8 & $E_{6(+6)} / S p(8 ; \mathbf{R})$ & $S L(6) / S O(6)$ & 20 & 10 \\
\hline 9 & $\frac{E_{6(+2)}}{S U(3,3) \times S U(1,1)}$ & $\frac{S U(3,3)}{S(U(3) \times U(3))}$ & 18 & 10 \\
\hline 10 & $\frac{E_{6(-14)}}{S O^{*}(10) \times S O(2)}$ & $S U(5,1) / U(5)$ & 10 & 10 \\
\hline 11 & $E_{7(+7)} / S U(4,4)$ & $\mathrm{SO}(6,6) / \mathrm{SO}(6) \times \mathrm{SO}(6)$ & 36 & 16 \\
\hline 12 & $\frac{E_{7(-5)}}{S O^{*}(12) \times S O(2,1)}$ & $S O^{*}(12) \times U(6)$ & 30 & 16 \\
\hline 13 & $\frac{E_{7(-25)}}{E_{6(-14)} \times S O(2)}$ & $\frac{S O(10,2)}{S O(10) \times S O(2)}$ & 20 & 16 \\
\hline 14 & $E_{8(+8)} / S O^{*}(16)$ & $E_{7(+7)} / S U(8)$ & 70 & 28 \\
\hline 15 & $\frac{E_{8(-24)}}{E_{7(-25)} \times S U(1,1)}$ & $\frac{E_{7(-25)}}{E_{6(-78)} \times S O(2)}$ & 54 & 28 \\
\hline
\end{tabular}

Apart from the trivial case $G / H=S L(2) / S O(2)$ with no scalars and no vectors we are left with 15 possibilities. They are listed in Table 2 together with the number of scalars, $\operatorname{dim} \bar{G} / \bar{H}$, and the number of vectors $k=\frac{1}{4} \operatorname{dim} G / H^{\prime \prime}$ for each case.

Due to the assumptions made in Steps 1-3 above, the adjoint representation of $G$ has the decomposition

$$
\operatorname{Ad} G=\operatorname{Ad} \bar{G} \otimes 1 \oplus \varrho \otimes 2 \oplus 1 \otimes 3
$$

into representations of $\bar{G} \times S L(2)$, where $\varrho$ is the $2 k$ dimensional real matrix representation describing the action (2.9) of $\bar{G}$ on the vector field strengths. This decomposition allows us to generalize the parametrization of the coset representatives $P(x)$ used by Julia [57] for $G=E_{8(+8)}$ to all the cases listed in Table 2. 
Table 3. Some static truncations of coset spaces appearing in Table 2

\begin{tabular}{|c|c|c|c|}
\hline$\#$ & $G_{\text {st }} / H_{\text {st }}$ & $\bar{G}_{\mathrm{st}} / \bar{H}_{\mathrm{st}}$ & $\operatorname{dim} \bar{G}_{\mathrm{st}} / \bar{H}_{\mathrm{st}}$ \\
\hline 1 & $G L(n+1) / S O(n, 1)$ & $G L(n) / S O(n)$ & $\frac{1}{2} n(n+1)$ \\
\hline 2 & $\frac{S O(p+1, q+1)}{S O(p, 1) \times S O(1, q)}$ & $S O(p, q) / S O(p) \times S O(q)$ & $p q$ \\
\hline 3 & $\frac{S O(p+1, q+1)}{S O(p, 1) \times S O(1, q)} \times \mathbf{R}$ & $\frac{S O(p, q)}{S O(p) \times S O(q)} \times \mathbf{R}$ & $p q+1$ \\
\hline 9 & $S p(8 ; \mathbf{R}) / G L(4)$ & $\frac{S O(3,3)}{S O(3) \times S O(3)}$ & 9 \\
\hline 10 & $U S p(4,4) / S p(4 ; \mathbf{C})$ & $S U^{*}(4) / U S p(4)$ & 5 \\
\hline $11 \mathrm{a}$ & $E_{6(+6)} / U S p(4,4)$ & $\frac{S O(5,5)}{S O(5) \times S O(5)}$ & 25 \\
\hline $11 b$ & $S L(8) / S O(4,4)$ & $\frac{S L(4) \times S L(4)}{S O(4) \times S O(4)}$ & 18 \\
\hline 12 & $\frac{S U(4,4)}{S L(4 ; \mathbf{C}) \times \mathbf{R}}$ & $S L(4 ; \mathbf{C}) / S U(4)$ & 15 \\
\hline 13 & $S U^{*}(8) / U S p(4,4)$ & $\frac{S U^{*}(4) \times S U^{*}(4)}{U S p(4) \times U S p(4)}$ & 10 \\
\hline 14 & $\mathrm{SO}(8,8) / \mathrm{SO}(8 ; \mathrm{C})$ & $S L(8) / S O(8)$ & 35 \\
\hline 15 & $S O^{*}(16) / S U(8) \times \mathbf{R}$ & $S U^{*}(8) / U S p(8)$ & 27 \\
\hline
\end{tabular}

Choosing the coefficient $\hat{c}=\frac{1}{2 k+4}$ for the adjoint representation leads to the scalar product (A.7) which reproduces the form (3.6) of the Lagrangian with the correct coefficients. Reversing the reduction from 4 to 3 dimensions we thus obtain a unique 4 dimensional theory for each case in our list.

In Sect. 2.2 we have defined the static truncation of the 4 dimensional $\bar{G} / \bar{H}$ $\sigma$-model which induces a corresponding static truncation of the 2 and 3 dimensional $G / H \sigma$-models. The precise form of these truncations depends on the assignment of temporal parities to the scalars and vectors. In Table 3 we give static truncations for some of the coset spaces listed in Table 2, all chosen such that the number of vector fields in the truncated theory, $k_{\mathrm{st}}=\frac{1}{2} \operatorname{dim} G_{\mathrm{st}} / H_{\mathrm{st}}^{\prime \prime}$, is the same as in the untruncated one.

\section{References}

1. Serini, R.: Rend. Lincei 27, 235 (1918)

2. Einstein, A., Pauli, W.: Ann. Math. 44, 131 (1943)

3. Lichnerowicz, A.: Théories Relativiste de la Gravitation et de l'Electromagnétisme. Paris: Masson 1955

4. Einstein, A., Rosen, N.: Phys. Rev. 48, 73 (1935)

5. Wheeler, J.A.: Geometrodynamics. New York: Academic Press 1962 
6. Black Holes. B. De Witt, C. De Witt (eds.), New York: Gordon and Breach 1973

7. Hawking, S.W., Israel, W.: General relativity. Cmabridge: Cambridge University Press 1979

8. Kerr, R.P.: Phys. Rev. Lett. 11, 237 (1963)

Newman, E.T., Couch, E., Chinapared, K., Exton, A., Prakash, A., Torrence, R.: J. Math. Phys. 6, 918 (1965)

Ernst, F.J.: Phys. Rev. 168, 1415 (1968)

9. Papapetrou, A.: Proc. Roy. Irish Acad. A 51, 191 (1947)

Majumdar, S.D.: Phys. Rev. 72, 390 (1947)

10. Schoen, R., Yau, S.T.: Commun. Math. Phys. 70, 231 (1981)

Witten, E.: Commun. Math. Phys. 80, 381 (1981)

11. Bunting, G.: Proof of the uniqueness conjecture for black holes. PHD thesis, Dept. of Math., University of New England, Armidale N.S.W. 1983

12. Mazur, P.O.: J. Phys. A 15, 3173 (1982)

13. B. Carter: In [6]

14. Bunting, G.L., Masood-Ul-Alaam, A.K.M.: Gen. Rel. Grav. 19, 147 (1987)

15. Israel, W.: Phys. Rev. 164, 1776 (1967)

16. Boucher, W., Gibbons, G.W., Horowitz, G.T.: Phys. Rev. D 30, 2447 (1984)

17. Hajicek, P.: Nucl. Phys. B 185, 254 (1981)

18. Gibbons, G.W., In: Unified theories of elementary particles. Breitenlohner, P., Dürr, H.P. (eds.), Berlin, Heidelberg, New York: Springer 1982

19. Gibbons, G.W., Hull, C.M.: Phys. Lett. 109 B, 190 (1982)

20. Harrison, B.K.: J. Math. Phys. 9, 1744 (1968)

21. Neugebauer, G., Kramer, D.: Ann. Phys. (Leipzig) 24, 62 (1969)

22. Geroch, R.: J. Math. Phys. 12, 918 (1971)

23. Kinnersley, W.: J. Math. Phys. 14, 651 (1973)

24. Belinskiî, V.A., Zakharov, V.E.: Zh. Eksp. Teor. Fiz. 75, 1955 (1978); 77, 3 (1979)

25. Maison, D.: Phys. Rev. Lett. 41, 521 (1978)

26. Geroch, R.: J. Math. Phys. 13, 394 (1972)

27. Solutions of Einstein's equations: techniques and results. Hoenselaers, C., Dietz, W. (eds.). Berlin, Heidelberg, New York: Springer 1984

28. Breitenlohner, P., Maison, D.: Ann. Inst. Henri Poincaré 46, 215 (1987)

29. Breitenlohner, P., Maison, D.: Generalized Geroch group for Kaluza-Klein theories (in preparation)

30. Helgason, S.: Differential geometry and symmetric spaces. New York: Academic Press 1962

31. Kobayashi, S., Nomizu, K.: Foundations of differential geometry. New York: Interscience 1969

32. Gaillard, M.K., Zumino, B.: Nucl. Phys. B 193, 221 (1981)

33. Simon, W.: J. Math. Phys. 25, 1038 (1984)

34. Hawking, S.W., Ellis, G.F.R.: The large scale structure of space-time. Cambridge: Cambridge University Press 1973

35. Colpi, M., Shapiro, S.L., Wasserman, I.: Phys. Rev. Lett. 57, 2485 (1986)

Wolf, C.: Phys. Lett. 117 A, 443 (1986)

36. McLenaghan, R.G., Tariq, N.: J. Math. Phys. 16, 2306 (1975)

37. Gannon, D.: J. Math. Phys. 16, 2364 (1975)

38. Ehlers, J.: Konstruktion und Charakterisierungen von Lösungen der Einsteinschen Gravitationsgleichungen. Dissertation, Hamburg 1957

39. Eells, Jr., J., Sampson, J.H.: Am. J. Math. 86, 109 (1964)

40. Dobiasch, P., Maison, D.: Gen. Rel. Grav. 14, 231 (1982)

41. Gibbons, G.W.: Nucl. Phys. B 207, 337 (1982)

42. Carter, B.: In: Gravitation in astrophysics. Proc. of the 1986 Cargèse Summerschool. Carter, B., Hartle, J.B. (eds.), New York: Plenum Press (to appear)

43. Robinson, D.C.: Gen. Rel. Grav. 8, 695 (1977)

44. Israel, W.: Commun. Math. Phys. 8, 245 (1968)

45. Maison, D.: In: Non-linear equations in classical and quantum field theory. Sanchez, N. (ed.), Berlin, Heidelberg, New York: Springer 1985 
46. Simon, W.: Gen. Rel. Grav. 17, 761 (1985)

47. Robinson, D.C.: Phys. Rev. Lett. 34, 905 (1975)

48. Mazur, P.O.: A global identity for nonlinear $\sigma$-models. Preprint, Department of Theor. Physics, Jagellonian University, Cracow, Oct. 1983

49. Breitenlohner, P., Maison, D.: Generalized Harrison transformations for black holes (in preparation)

50. Gibbons, G.W., Ruback, P.J.: Phys. Rev. Lett. 57, 1492 (1986)

51. Perjes, Z.: Phys. Rev. Lett. 27, 1668 (1971) Israel, W., Wilson, G.A.: J. Math. Phys. 13, 865 (1972)

52. Hartle, J.B., Hawking, S.W.: Commun. Math. Phys. 26, 87 (1972)

53. Kramer, D., Neugebauer, G.: Phys. Lett. 75 A, 259 (1980)

54. Bekenstein, J.: Phys. Rev. D 7, 2333 (1973)

55. Hiscock, W.A.: J. Math. Phys. 22, 1828 (1981)

56. Gilmore, R.: Lie groups, Lie algebras and their applications. New York: Wiley 1974

57. Julia, B.: In [58]

58. In: Unified field theories of more than 4 dimensions. De Sabbata, V., Schmutzer, E. (eds.), Singapore: World Scientific 1983

59. Eichenherr, H., Forger, M.: Nucl. Phys. B 155, 382 (1979)

Communicated by S.-T. Yau

Received April 17, 1987; in revised form July 29, 1988 
